\title{
UNIVERSAL LOG STRUCTURES ON SEMI-STABLE VARIETIES
}

\author{
MARTIN C. OLSSON
}

\begin{abstract}
Fix a morphism of schemes $f: \underline{X} \rightarrow \underline{S}$ which is flat, proper, and "fiber-by-fiber semi-stable". Let $I V L S$ be the functor on the category of log schemes over $\underline{S}$ which to any $T$ associates the isomorphism classes of pairs $\left(\mathcal{M}_{X}, f^{b}\right)$, where $\mathcal{M}_{X}$ is a log structure on $\underline{X} \times_{S} \underline{T}$ and $f^{b}: p r_{2}^{*} \mathcal{M}_{T} \rightarrow \mathcal{M}_{X}$ is a morphism of log structures making $\left(\underline{X} \times \underline{S} \underline{T}, \mathcal{M}_{X}\right) \rightarrow T$ a log smooth, integral, and vertical morphism. The main result of this paper is that $I V L S$ is representable by a log scheme. In the course of the proof we also generalize results of F. Kato on the existence of log structures of embedding and semi-stable type.
\end{abstract}

\section{INTRODUCTION}

In light of the powerful tools of logarithmic geometry developed in recent years, an important question to ask when given a singular morphism $f$ : $\underline{X} \rightarrow \underline{S}$ of schemes is whether there exist fine log structures on $\underline{X}$ and $\underline{S}$ making $f$ a $\log$ smooth morphism. In addition, if such log structures exist one would like to know to what extent they are unique. This paper is an attempt to understand the situation for morphisms which are fiber-by-fiber semi-stable (see below for the precise definition). Our inspiration comes from the well-understood case of semi-stable curves, and the main result of this paper can be viewed as a generalization of the statement that the Deligne-Mumford compactification of the moduli space of curves of genus $g>1$ is a moduli stack for certain log curves ([11]). This work was heavily influenced by the papers of F. Kato ([10], [11]).

Let $f: \underline{X} \rightarrow \underline{S}$ be a flat and proper morphism of schemes of finite type over an excellent Dedekind ring, and suppose that for every geometric point $\bar{s} \rightarrow \underline{S}$ the fiber $\underline{X}_{\bar{s}}$ is a semi-stable variety in the sense of the following definition:

The contents of this paper grew out of a portion of the author's 2001 dissertation written under the supervision of Professor A. Ogus at UC Berkeley. The author thanks Professor Ogus for his very helpful advice and suggestions. This research was partially conducted by the author for the Clay Mathematics Institute. 
Definition 1.1. A scheme $\underline{Y} / k$ over a separably closed field is a semistable variety if for each closed point $y \in \underline{Y}$ there exists an étale neighborhood $\left(\underline{U}, y^{\prime}\right)$ of $y$, integers $r \geq l$, and an étale morphism

$$
\underline{U} \longrightarrow k\left[X_{1}, \ldots, X_{r}\right] /\left(X_{1} \cdots X_{l}\right),
$$

sending $y^{\prime}$ to the point $X_{1}=\cdots=X_{r}=0$ (we make the convention that if $l=0$ then $\left.\left(X_{1} \cdots X_{l}\right)=(0)\right)$.

Let $F \log$ denote the category of fine $\log$ schemes over $\underline{S}$, and define the functor of integral and vertical log structures

$$
\text { IVLS : FLog } \longrightarrow \text { Set }
$$

as follows: for any fine $\log$ scheme $T$ over $\underline{S}, I V L S(T)$ is the set of isomorphism classes of pairs $\left(\mathcal{M}_{X_{T}}, f_{T}^{b}\right)$, where $\mathcal{M}_{X_{T}}$ is a fine log structure on $\underline{X}_{T}:=\underline{X} \times_{\underline{S}} \underline{T}$ and $f_{T}^{b}: p r_{2}^{*} \mathcal{M}_{T} \rightarrow \mathcal{M}_{X_{T}}$ is a morphism of log structures making

$$
\left(p r_{2}, f_{T}^{b}\right):\left(\underline{X}_{T}, \mathcal{M}_{X_{T}}\right) \longrightarrow T
$$

a log smooth, vertical, and integral morphism (recall that a morphism is vertical if $\mathcal{M}_{X_{T} / T}:=\operatorname{Cok}\left(p_{2}^{*} \mathcal{M}_{T} \rightarrow \mathcal{M}_{X_{T}}\right)$ is a sheaf of groups). The main result of this paper is the following theorem:

Theorem 1.2. The functor IVLS is representable by a log scheme, and the map on underlying schemes $\underline{I V L S} \rightarrow \underline{S}$ is a monomorphism of finite type.

The paper is organized as follows.

Section 2 is devoted to showing that if $f: X \rightarrow S$ is a proper, log smooth, integral, and vertical morphism all of whose geometric fibers are semi-stable varieties, then there exists a canonical cartesian diagram

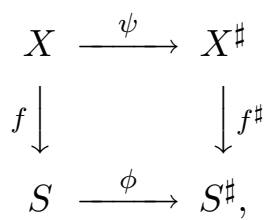

where $f^{\sharp}: X^{\sharp} \rightarrow S^{\sharp}$ has a special form and the underlying maps of schemes of $\phi$ and $\psi$ are isomorphisms. This result will be used to show that the functor $I V L S$ is equivalent to the functor defined by a pair $\left(F, \mathcal{M}_{F}\right)$, where $F$ is a functor on the category of $\underline{S}$-schemes and $\mathcal{M}_{F}$ is a "log structure" on $F$.

More precisely, let $\log _{\underline{S}}$ denote the fibered category over the category of $\underline{S}$-schemes whose fiber over an $\underline{S}$-scheme $\underline{T}$ is the groupoid of fine log structures $\mathcal{M}_{T}$ on $\underline{T}$. It is shown in $([16], 1.1)$ that $\mathcal{L}_{\log }$ is an algebraic stack. By definition, a $\log$ structure on a fibered category $\mathcal{S}$ is a morphism of fibered categories $\mathcal{S} \rightarrow \mathcal{L}_{o} g_{\underline{S}}$. Viewing functors on the category of $\underline{S}$-schemes as fibered categories, we get a notion of a $\log$ structure on a functor. 
Now suppose $\left(F, \mathcal{M}_{F}\right)$ is a functor with a $\log$ structure. Then for any element $t \in F(\underline{T})$ over some $\underline{S}$-scheme $\underline{T}$, we get a $\log$ structure $t^{*} \mathcal{M}_{F}$ on $\underline{T}$ by viewing $t$ as a morphism of functors $\underline{T} \rightarrow F$ and defining $t^{*} \mathcal{M}_{F}$ to be the $\log$ structure obtained from the composite $\mathcal{M}_{F} \circ t: \underline{T} \rightarrow \mathcal{L}_{o g}$. This enables us to define a functor $F^{l o g}$ on $F \log$ as follows: for any $T=\left(\underline{T}, \mathcal{M}_{T}\right)$, $F^{\log }(T)$ is the set of pairs $\left(t, t^{b}\right)$, where $t \in F(\underline{T})$ and $t^{b}: t^{*} \mathcal{M}_{F} \rightarrow \mathcal{M}_{T}$ is a morphism of $\log$ structures. To say that $I V L S$ is equivalent to the functor defined by a pair $\left(F, \mathcal{M}_{F}\right)$ means that there exists an isomorphism of functors $I V L S \simeq F^{l o g}$. The importance of this is that the proof of theorem 1.2 is reduced to showing that the (ordinary) functor $F$ is representable by a scheme, and that $F \rightarrow \underline{S}$ is a monomorphism of finite type.

In section 3 we generalize results of F. Kato ([10]) on the existence of log structures of semi-stable and embedding type (see the text for definitions) to an arbitrary base scheme. Our generalization of F. Kato's work includes cohomological obstructions for the existence of log structures of semi-stable and embedding type and so might also be of some interest for schemes over fields. This section can be read independently from the rest of the paper.

In section 4 we use the results of sections 2 and 3 to prove a theorem about effectivity of certain formal log schemes. This theorem will be used in section 5 .

Finally in section 5 we bring it all together to prove theorem 1.2. The proof is based on M. Artin's method ([5], 5.4) and the results of ([16], [17]).

1.1. Conventions. Throughout this paper we denote schemes by underlined letters (e.g. $\underline{X}$ ) and $\log$ schemes by unadorned letters (e.g. $X)$. For a $\log$ scheme $X$, we denote the underlying scheme by $\underline{X}$. The reader is assumed to be familiar with logarithmic geometry at the level of $([9],[12]$, or [16]). Our conventions about algebraic stacks are those of ([13]), except we only assume that our stacks are locally quasi-separated and not necessarily quasi-separated ( $\log _{S}$ is not quasi-separated).

\section{Special elements of $I V L S$}

Let $f: X \rightarrow S$ be a smooth, proper, integral, and vertical morphism of noetherian log schemes, and suppose that for every geometric point $\bar{s} \rightarrow \underline{S}$, the underlying scheme of $X_{\bar{s}}$ is a semi-stable variety (in the sense of definition 1.1). The purpose of this section is to show that under these assumptions the $\log$ structure $\mathcal{M}_{X}$ on $\underline{X}$ is induced by base change from a smooth morphism

$$
f^{\sharp}: X^{\sharp} \rightarrow S^{\sharp}
$$

of a special form whose underlying morphism of schemes is that of the original $f$. 
Definition 2.1. A $\log$ smooth morphism $f: X \rightarrow S$ is essentially semistable if for each geometric point $\bar{x} \rightarrow \underline{X}$ the monoids $\left(f^{-1} \overline{\mathcal{M}}_{S}\right)_{\bar{x}}$ and $\overline{\mathcal{M}}_{X, \bar{x}}$ are free monoids, and if for suitable isomorphisms $\left(f^{-1} \overline{\mathcal{M}}_{S}\right)_{\bar{x}} \simeq \mathbb{N}^{r}$ and $\overline{\mathcal{M}}_{X, \bar{x}} \simeq \mathbb{N}^{r+s}$ the map

$$
\left(f^{-1} \overline{\mathcal{M}}_{S}\right)_{\bar{x}} \rightarrow \overline{\mathcal{M}}_{X, \bar{x}}
$$

is of the form

$$
e_{i} \mapsto\left\{\begin{array}{cc}
e_{i} & \text { if } i \neq r \\
e_{r}+e_{r+1}+\cdots+e_{r+s} & \text { if } i=r,
\end{array}\right.
$$

where $e_{i}$ denotes the $i$-th standard generator of $\mathbb{N}^{r}$.

Lemma 2.2. If $f: X \rightarrow S$ is essentially semi-stable, then étale locally on $X$ and $S$ there exist charts $\mathbb{N}^{r} \rightarrow \mathcal{M}_{S}, \mathbb{N}^{r+s} \rightarrow \mathcal{M}_{X}$ such that the map $\mathbb{N}^{r} \rightarrow \mathbb{N}^{r+s}$ given by formula 2.1 is a chart for $f$, and such that the map

$$
\mathcal{O}_{S} \otimes_{\mathbb{Z}\left[\mathbb{N}^{r}\right]} \mathbb{Z}\left[\mathbb{N}^{r+s}\right] \rightarrow \mathcal{O}_{X}
$$

is smooth.

Proof. Observe that if $s \in \underline{S}$ is a point, then the stalk $\overline{\mathcal{M}}_{S, \bar{s}}$ is a free monoid and hence in some étale neighborhood of $s$ there exists a chart $\mathbb{N}^{r} \rightarrow \mathcal{M}_{S}$ such that the induced map $\mathbb{N}^{r} \rightarrow \overline{\mathcal{M}}_{S, \bar{s}}$ is bijective. If $x \in \underline{X}$ is a point lying over $s$, then by $([15], 2.25)$ there exists in some étale neighborhood of $x$ a chart

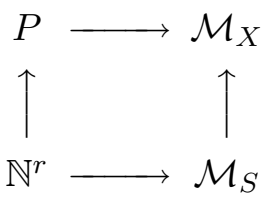

such that the induced map

$$
\mathcal{O}_{S} \otimes_{\mathbb{Z}[\mathbb{N} r]} \mathbb{Z}[P] \rightarrow \mathcal{O}_{X}
$$

is smooth and such that the map $P \rightarrow \overline{\mathcal{M}}_{X, \bar{x}}$ is bijective. From the bijectivity of $P \rightarrow \overline{\mathcal{M}}_{X, \bar{x}}$ we conclude that $P$ is a free monoid, and that the map $\mathbb{N}^{r} \rightarrow P$ has the desired form (after perhaps applying an automorphism of $\left.\mathbb{N}^{r}\right)$.

Lemma 2.3. An essentially semi-stable morphism $f: X \rightarrow S$ is integral and vertical.

Proof. To see the integrality we have to check that the map $\mathbb{N}^{r} \rightarrow \mathbb{N}^{r+s}$ described in formula 2.1 is integral. For this observe that if $\Delta: \mathbb{N} \rightarrow \mathbb{N}^{s+1}$ is the diagonal map, then there exists a natural cocartesian diagram

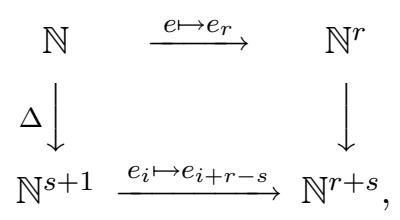


and since the map $\Delta$ is integral so is the map $\mathbb{N}^{r} \rightarrow \mathbb{N}^{r+s}$ ([12], 4.1 (i)). To see that it is vertical, let $Q$ be the cokernel of $\mathbb{N}^{r} \rightarrow \mathbb{N}^{r+s}$. By definition of cokernel, to give a map $Q \rightarrow M$ to an integral monoid $M$ is equivalent to giving a map $\mathbb{N}^{r+s} \rightarrow M$ such that the composite $\mathbb{N}^{r} \rightarrow \mathbb{N}^{r+s} \rightarrow M$ is zero. A map $\mathbb{N}^{r+s} \rightarrow M$ is equivalent to giving elements

$$
m_{1}, \ldots, m_{r+s} \in M
$$

and the condition that the map $\mathbb{N}^{r} \rightarrow M$ be zero means that

$$
m_{1}=m_{2}=\cdots=m_{r-1}=m_{r}+\cdots+m_{r+s}=0 .
$$

Thus giving an arrow $Q \rightarrow M$ is equivalent to giving elements $m_{r}, \ldots, m_{r+s}$ in $M^{*}$ such that $m_{r}+\cdots+m_{r+s}=0$. This in turn is equivalent to giving elements $m_{r}, \ldots, m_{r+s-1} \in M^{*}$. But the functor on the category of integral monoids

$$
M \mapsto\left\{r+s-1 \text {-tuples of elements } m \in M^{*}\right\}
$$

is represented by $\mathbb{Z}^{r+s-1}$, and hence $Q \simeq \mathbb{Z}^{r+s-1}$.

Recall that if $P$ is a sharp monoid (this means that $P^{*}=\{0\}$ ), then an element $p \in P-\{0\}$ is irreducible if for any equality $p_{1}+p_{2}=p$ in $P$ we have $p_{1}=0$ or $p_{2}=0$. The basic results about irreducible elements is the following proposition whose proof we omit (see [9], I.2.1.2):

Proposition 2.4. If $P$ is a fine sharp monoid, then the set $\operatorname{Irr}(P)$ of irreducible elements in $P$ is a finite set which generates $P$.

Suppose $\underline{S}=\operatorname{Spec}(k)$, where $k$ is a separably closed field, and that $f$ : $X \rightarrow S$ is essentially semi-stable. Let $x \in \underline{X}$ be a singular point. Then by lemma 2.2 there exists a chart

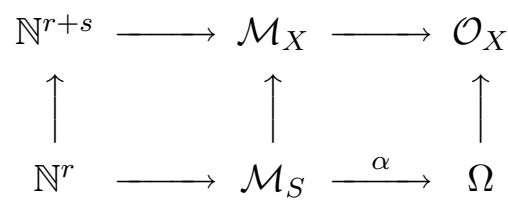

in an étale neighborhood of $x$ such that

$$
k \otimes_{\mathbb{Z}\left[\mathbb{N}^{r}\right]} \mathbb{Z}\left[\mathbb{N}^{r+s}\right] \simeq k\left[x_{r}, \ldots, x_{r+s}\right] /\left(x_{r} \cdots x_{r+s}-\alpha\left(e_{r}\right)\right) \rightarrow \mathcal{O}_{X}
$$

is smooth. Since $x$ is a singular point it follows that $\alpha\left(e_{r}\right)=0$ and hence the map

$$
\overline{\mathcal{M}}_{S} \rightarrow \overline{\mathcal{M}}_{X, \bar{x}}
$$

is of the form $\mathbb{N}^{r^{\prime}} \rightarrow \mathbb{N}^{r^{\prime}+s}$ as in definition 2.1 for some $r^{\prime} \leq r$. It follows that there is a unique element in the set of irreducible elements $\operatorname{Irr}\left(\overline{\mathcal{M}}_{S}\right)$ of $\overline{\mathcal{M}}_{S}$ whose image in $\overline{\mathcal{M}}_{X, \bar{x}}$ is not irreducible. This defines a canonical map

$$
s_{X}:\{\text { singular points of } X\} \rightarrow \operatorname{Irr}\left(\overline{\mathcal{M}}_{S}\right) .
$$


Definition 2.5. An essentially semi-stable morphism of $\log$ schemes $f$ : $X \rightarrow S$ is special at a geometric point $\bar{s}$ if the map

$$
s_{X_{\bar{s}}}:\left\{\text { singular points of } X_{\bar{s}}\right\} \rightarrow \operatorname{Irr}\left(\overline{\mathcal{M}}_{S, \bar{s}}\right)
$$

induces a bijection between the set of connected components of the singular locus of $X_{\bar{s}}$ and $\operatorname{Irr}\left(\overline{\mathcal{M}}_{S, \bar{s}}\right)$. If $f$ is special at every geometric point $\bar{s} \rightarrow \underline{S}$, then we call $f$ a special morphism.

Theorem 2.6. Let $f: X \rightarrow S$ be a smooth, proper, integral, and vertical morphism of noetherian log schemes, and suppose that for every geometric point $\bar{s} \rightarrow \underline{S}$ the underlying scheme of $X_{\bar{s}}$ is a semi-stable variety. Then there exists a pair of log structures $\left(\mathcal{M}_{S}^{\sharp}, \mathcal{M}_{X}^{\sharp}\right)$ on $\underline{S}$ and $\underline{X}$ respectively and a morphism

$$
f^{\sharp}: X^{\sharp}=\left(\underline{X}, \mathcal{M}_{X}^{\sharp}\right) \rightarrow S^{\sharp}=\left(\underline{S}, \mathcal{M}_{S}^{\sharp}\right)
$$

which is special, together with morphisms of log structures

$$
\phi: \mathcal{M}_{S}^{\sharp} \rightarrow \mathcal{M}_{S}, \quad \psi: \mathcal{M}_{X}^{\sharp} \rightarrow \mathcal{M}_{X}
$$

which make the diagram

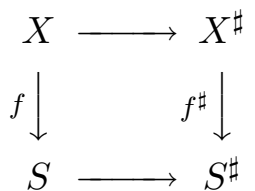

cartesian. Moreover, the datum $\left(\mathcal{M}_{S}^{\sharp}, \mathcal{M}_{X}^{\sharp}, \phi, \psi\right)$ is unique up to unique isomorphism.

The proof occupies the remainder of this section.

Let us begin by considering the case when $\underline{S}$ is the spectrum of a strictly henselian local ring $\mathcal{O}$, and $\mathcal{M}_{S}$ admits a chart $Q \rightarrow \mathcal{M}_{S}$ such that $Q \rightarrow$ $\overline{\mathcal{M}}_{S, s_{0}}$ is bijective (here $s_{0}$ denotes the closed point). Denote by $T$ the set of connected components of the singular locus of the closed fiber.

Recall ([9], I.1.1) that if $P$ is a monoid, then a congruence relation on $P$ is a subset $E \subset P \oplus P$ which is both a submonoid and a set-theoretic equivalence relation. A subset $S \subset E$ generates the congruence relation $E$ if $E$ is the smallest congruence relation on $P$ containing $S$. For any equivalence relation $E$ on $P$, the surjection $P \rightarrow P / E$ induces a structure of a monoid on $P / E$ if and only if $E$ is a congruence relation. Therefore, there is a natural bijection between isomorphism classes of surjective maps of monoids $P \rightarrow P^{\prime}$ and the set of congruence relations on $P$.

Lemma 2.7. Let $P$ be a fine sharp monoid, $Q \hookrightarrow P$ an integral morphism (such a morphism is automatically injective by [12], paragraph preceding 4.7), and let $\pi: P \rightarrow P / Q$ be the projection map.

1. For each $i \in P / Q$, there exists a unique element $p_{i} \in P$ such that 


$$
\pi^{-1}(i)=\left\{p_{i}+q \mid q \in Q\right\} .
$$

2. If $p \in P$ is an irreducible element not in $Q$ and if $i=\pi(p)$, then $p=p_{i}$ (notation is in part 1).

3. If $\left\{p_{i_{1}}, \ldots, p_{i_{n}}\right\}$ denotes the set of irreducible elements of $P$ which are not in $Q$ (we index these irreducible elements by their images in $P / Q$ ), then the congruence relation on $Q \oplus \mathbb{N}^{n}$ defined by the surjection

$$
Q \oplus \mathbb{N}^{n} \longrightarrow P, \quad(q,(\underline{n})) \mapsto q+\sum_{j=1}^{n} n_{j} p_{i_{j}}
$$

is generated by equalities

$$
\sum_{j=1}^{n} m_{j} p_{i_{j}}=q+\sum_{j=1}^{n} n_{j} p_{i_{j}}
$$

in $P$, where for each $j$ either $m_{j}$ or $n_{j}$ is equal to 0.

Proof. 1. Since $P$ is fine and sharp, there exists a partial ordering on $P^{+}$ defined by $p_{1} \leq p_{2}$ if there exists $p_{3} \in P$ such that $p_{1}+p_{3}=p_{2}$. By ([9], I.2.1.5.3) the set $\pi^{-1}(i)$ contains a unique finite set of minimal elements with respect to this partial ordering.

Suppose $p_{1}, p_{2} \in \pi^{-1}(i)$ and suppose $p_{1}$ is a minimal element. By construction of the quotient, there exists $q_{1}, q_{2} \in Q$ such that $p_{1}+q_{1}=p_{2}+q_{2}$. Since the map is integral, there exists $q_{3}, q_{4} \in Q$ and $p \in P$ such that

$$
p_{1}=q_{3}+p, \quad p_{2}=q_{4}+p, \quad q_{1}+q_{3}=q_{2}+q_{4} .
$$

By definition of quotient, $p \in \pi^{-1}(i)$ and $p \leq p_{1}$. Since $p_{1}$ was minimal it follows that $p_{1}=p$ and that $p_{2}=p_{1}+q_{4}$.

2. By definition of $p_{i}$, there exists $q \in Q$ such that $p=p_{i}+q$. Since $p$ is irreducible and has non-zero image in $P / Q$ this means that $q=0$.

3. Since $\operatorname{Irr}(P)$ generates $P$, the congruence relation is generated by equalities

$$
q+\sum_{j} n_{i_{j}} p_{i_{j}}=q^{\prime}+\sum_{j} m_{i_{j}} p_{i_{j}}
$$

in $P$. By the integrality of $Q \rightarrow P$, for any such equality there exists $p \in P$ and $q_{3}, q_{4} \in Q$ such that

$$
\sum_{j} n_{i_{j}} p_{i_{j}}=p+q_{3}, \quad \sum_{j} m_{i_{j}} p_{i_{j}}=p+q_{4}, \quad q+q_{3}=q+q_{4} .
$$

In addition, writing $p=\sum b_{i_{j}} p_{i_{j}}+q_{5}$ we see that the congruence relation on $Q \oplus \mathbb{N}^{n}$ defined by the surjection

$$
Q \oplus \mathbb{N}^{n} \rightarrow P
$$


is generated by equalities in $P$ of the form

$$
\sum_{j} n_{i_{j}} p_{i_{j}}=\sum_{j} b_{i_{j}} p_{i_{j}}+q .
$$

Moreover, since $P$ is cancellative we can assume that for each $j$ either $n_{i_{j}}=0$ or $b_{i_{j}}=0$.

Corollary 2.8. Let $t$ be a singular point of $X$, and let $n \in \mathcal{M}_{X / S, \bar{t}}$. Then there exists a unique element $p_{n} \in \overline{\mathcal{M}}_{X, \bar{t}}$ such that

$$
\pi^{-1}(n)=\left\{p_{n}+q \mid q \in \overline{\mathcal{M}}_{S, s_{0}}\right\} .
$$

Proof. The map $\overline{\mathcal{M}}_{S, s_{0}} \rightarrow \overline{\mathcal{M}}_{X, \bar{t}}$ is integral by assumption.

As above let $s_{0}$ denote the closed point of $S$, and let $X_{s_{0}}$ denote the fiber. By ([15], 2.25), for every singular closed point $t$ of $X_{s_{0}}$ there exists a chart

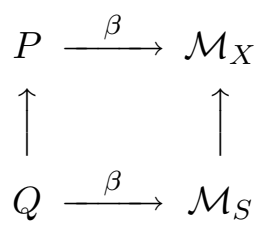

in some étale neighborhood of $t$ such that

(1) $P \rightarrow \overline{\mathcal{M}}_{X, \bar{t}}$ is bijective.

(2) The map

$$
\mathcal{O} \otimes_{\mathbb{Z}[Q]} \mathbb{Z}[P] \rightarrow \mathcal{O}_{X}
$$

is smooth.

Let $\left\{p_{i_{1}}, \ldots, p_{i_{n}}\right\}$ be the set of irreducible elements in $P$ which are not in $Q$ (as in lemma 2.7 we index these irreducible elements by their images in $P / Q)$. If $i \in P / Q$ is any element and $p_{i}$ is as in 2.7 , then $p_{i}$ can be written as

$$
p_{i}=\sum m_{j} p_{i_{j}} .
$$

Indeed writing $p_{i}$ as a sum of irreducible elements we have

$$
p_{i}=\sum m_{j} p_{i_{j}}+q, \quad q \in Q
$$

and by definition of $p_{i}$ we must have $q=0$. Hence the map

$$
\gamma: \mathcal{O}\left[Y_{i_{1}}, \ldots, Y_{i_{n}}\right] \rightarrow \mathcal{O} \otimes_{\mathbb{Z}[Q]} \mathbb{Z}[P], \quad Y_{i_{j}} \mapsto 1 \otimes e\left(p_{i_{j}}\right)
$$

is surjective. Let $J$ be the kernel of $\gamma$.

Proposition 2.9. $\quad$ (1) There exists an element $q_{0} \in Q$ such that

$$
\sum_{j=1}^{n} p_{i_{j}}=q_{0} .
$$


(2) $J$ is generated by the element

$$
\prod_{j=1}^{n} Y_{i_{j}}-\alpha\left(q_{0}\right) .
$$

(3) Let $Q \oplus_{\mathbb{N}} \mathbb{N}^{n}$ be the pushout of the diagram

$$
\begin{gathered}
\stackrel{\Delta}{\substack{1 \mapsto q_{0} \\
\downarrow}} \mathbb{N}^{n} \\
Q .
\end{gathered}
$$

Then the natural map

$$
Q \oplus_{\mathbb{N}} \mathbb{N}^{n} \rightarrow P, \quad\left(q,\left(\underline{n}_{i_{j}}\right)\right) \mapsto q+\sum n_{i_{j}} p_{i_{j}}
$$

is an isomorphism.

Proof. We first reduce to the case when $\mathcal{O}=k\left(s_{0}\right)$. The reduction step is trivial for (1) and (3). Let us show that (2) holds if the proposition is true on the closed fiber. Since

$$
\sum_{j=1}^{n} p_{i_{j}}=q_{0}, \quad q_{0} \in Q
$$

there is an inclusion of ideals

$$
\left(\prod Y_{i_{j}}-\alpha\left(q_{0}\right)\right) \subset J
$$

which defines a closed immersion

$$
\operatorname{Spec}\left(\mathcal{O}\left[Y_{i_{1}}, \ldots, Y_{i_{n}}\right] / J\right) \hookrightarrow \operatorname{Spec}\left(\mathcal{O}\left[Y_{i_{1}}, \ldots, Y_{i_{n}}\right] /\left(\prod Y_{i_{j}}-\alpha\left(q_{0}\right)\right) .\right.
$$

Since both of these schemes are flat over $\mathcal{O}$ and the reduction is an isomorphism, the closed immersion is an isomorphism also by ([1], VII.1.9). Therefore (2) holds and hence it suffices to consider the case when $\mathcal{O}=k$ for some separably closed field $k$.

Lemma 2.10. The ideal $J=\operatorname{Ker}(\gamma)$ is generated by elements of the form

$$
\prod_{j} Y_{i_{j}}^{n_{i_{j}}}-\alpha(q) \prod_{j} Y_{i_{j}}^{m_{i_{j}}}
$$

where

$$
\sum_{j} n_{i_{j}} p_{i_{j}}=\sum_{j} m_{i_{j}} p_{i_{j}}+q, \quad q \in Q
$$

in $P$ and for each $j$ either $n_{i_{j}}$ or $m_{i_{j}}$ is equal to 0 .

Proof. This is a restatement of 2.7.3. Indeed it suffices to show that the kernel of the map

$$
\mathbb{Z}\left[Q \oplus \mathbb{N}^{n}\right] \rightarrow \mathbb{Z}[P]
$$


induced by the surjection

$$
Q \oplus \mathbb{N}^{n} \rightarrow P, \quad\left(q,\left(\underline{n}_{j}\right)\right) \mapsto q+\sum_{j} n_{j} p_{i_{j}}
$$

is the ideal defined by any set of generators for the congruence relation on $Q \oplus \mathbb{N}^{n}$. This follows from the universal property of monoid algebras: for any algebra $R$, there is a natural bijection

$$
\mathrm{Hom}_{\mathrm{Alg}}(\mathbb{Z}[P], R) \longrightarrow \mathrm{Hom}_{M o n}(P, R)
$$

where $R$ is viewed as a multiplicative monoid.

Lemma 2.11. (1) The completion $\widehat{\mathcal{O}}_{X, t}$ is isomorphic to

$$
k\left[\left[T_{1}, \ldots, T_{s}, Y_{i_{1}}, \ldots, Y_{i_{n}}\right]\right] /\left(Y_{i_{1}} \cdots Y_{i_{n}}\right)
$$

where $\left\{T_{1}, \ldots, T_{s}\right\}$ are independent variables.

(2) Let $L \subset P$ be the submonoid generated by the set $\left\{p_{i} \mid i \in P / Q\right\}$. Then $L$ is isomorphic to $\mathbb{N}^{n}$.

Proof. Let $Y^{\prime}$ be the log scheme with underlying scheme $\operatorname{Spec}\left(k \otimes_{\mathbb{Z}[Q]} \mathbb{Z}[P]\right)$ and $\log$ structure induced by $P \rightarrow \mathbb{Z}[P]$. Then there exists a diagram of log schemes

$$
X \stackrel{h}{\longrightarrow} Y^{\prime} \longrightarrow S
$$

where $h$ is $\log$ smooth and strict. Since $h$ is strict, the map

$$
\Omega_{\underline{X} / \underline{S}}^{1} \rightarrow \Omega_{X / Y^{\prime}}^{1}
$$

is surjective, so in some neighborhood of $t$ there exist elements $f_{1}, \ldots, f_{s}$ in the maximal ideal of $t$ such that $d f_{1}, \ldots, d f_{s}$ map to a basis for $\Omega_{X / Y^{\prime}}^{1} \otimes k(t)$. This defines a map

$$
h^{\prime}: X \rightarrow Y^{\prime} \times \underline{S} \mathbb{A}_{\underline{S}}^{S}
$$

of $\log$ schemes which is strict, and for which the map

$$
h^{\prime *} \Omega_{Y^{\prime} \otimes_{\underline{S}} \mathbb{A}_{\underline{S}}^{s} / Y^{\prime}} \otimes k(t) \rightarrow \Omega_{X / Y^{\prime}}^{1} \otimes k(t)
$$

is an isomorphism. It follows that in some neighborhood of $t$ the map $h^{\prime}$ is étale $([15], 2.20)$.

Thus we can extend the map $\gamma$ to an étale map

$$
X \rightarrow \operatorname{Spec}\left(k\left[T_{1}, \ldots, T_{s}\right] \otimes_{k}\left(k\left[Y_{i_{1}}, \ldots, Y_{i_{n}}\right] / J\right)\right)
$$

sending $t$ to the point $T_{1}=\cdots=T_{s}=Y_{i_{1}}=\cdots=Y_{i_{n}}=0$.

First we claim that the resulting map

$$
k\left[\left[T_{1}, \ldots, T_{s}, Y_{i_{1}}, \ldots, Y_{i_{n}}\right]\right] \rightarrow \widehat{\mathcal{O}}_{X, t}
$$

induces an isomorphism

$$
k\left[\left[T_{1}, \ldots, T_{s}, Y_{i_{1}}, \ldots, Y_{i_{n}}\right]\right] /\left(T_{1}, \ldots, T_{s}, Y_{i_{1}}, \ldots, Y_{i_{n}}\right)^{2} \simeq \widehat{\mathcal{O}}_{X, t} / \mathfrak{m}^{2} .
$$


To see this it suffices to show that a set of defining equations for $J$ are contained in the ideal $\left(Y_{i_{1}}, \ldots, Y_{i_{n}}\right)^{2}$. But $J$ is generated by equations arising from non-trivial equalities

$$
\sum n_{i_{j}} p_{i_{j}}=q+\sum m_{j} p_{i_{j}}
$$

in $P$. If $\sum n_{i_{j}}=1$, then the equality is

$$
p_{i_{0}}=q+\sum m_{j} p_{i_{j}}
$$

for some $j_{0}$. By the irreducibility of $p_{i_{j}}$, this implies that the equation comes from an equality

$$
p_{i_{j}}=q \quad \text { or } \quad p_{i_{j}}=p_{i_{j^{\prime}}} .
$$

Since $p_{i_{j}} \notin Q$ we must have $p_{i_{j}}=p_{i_{j^{\prime}}}$ and hence the equation is trivial.

Fix an isomorphism

$$
\widehat{\mathcal{O}}_{X, t} \simeq k\left[\left[X_{1}, \ldots, X_{l}\right]\right] /\left(X_{1} \cdots X_{h}\right)
$$

for some $l$ and $h$ (this is possible by assumption), and let $\mathfrak{m}$ denote the maximal ideal of $\widehat{\mathcal{O}}_{X, t}$. It follows from the above that

$$
l=\operatorname{dim}\left(\mathfrak{m} / \mathfrak{m}^{2}\right)=n+s .
$$

For each $k \geq 1$, the dimension of $\mathfrak{m}^{k} / \mathfrak{m}^{k+1}$ is equal to the number of monomials of degree $k$ in $X_{1}, \ldots, X_{l}$ which do not contain the string $X_{1} \cdots X_{h}$. In particular, the dimension of $\mathfrak{m}^{h} / \mathfrak{m}^{h+1}$ is equal to one less than the number of monomials of degree $h$ in $l=n+s$ variables. This implies that we have at least one equation involving monomials in the $Y_{i_{j}}$ of degree $h$. Fix one such equation $f(\underline{Y})=0$. By lemma 2.10, we can choose our equation so that it is either of the form

$$
f(\underline{Y})=Y_{i_{1}} \cdots Y_{i_{h}}-Y_{i_{1}^{\prime}} \cdots Y_{i_{m}^{\prime}}
$$

or of the form

$$
f(\underline{Y})=Y_{i_{1}} \cdots Y_{i_{h}} .
$$

Let $R$ be the ring

$$
k\left[\left[T_{1}, \ldots, T_{s}, Y_{i_{1}}, \ldots, Y_{i_{n}}\right]\right] /(f),
$$

and let $\psi: R \rightarrow \widehat{\mathcal{O}}_{X, t}$ be the natural map.

Sub-Lemma 2.12. Let $\psi: A \rightarrow B$ be a surjective local homomorphism between complete noetherian local rings. If the induced map

$$
g r^{*}(\psi): g r_{\mathfrak{m}_{A}}^{*}(A) \rightarrow g r_{\mathfrak{m}_{B}}^{*}(B)
$$

is an isomorphism, then $\psi$ is an isomorphism.

Proof. Because $A$ and $B$ are complete with respect to $\mathfrak{m}_{A}$, it suffices to show that $A / \mathfrak{m}_{A}^{n} \rightarrow B / \mathfrak{m}_{B}^{n}$ is an isomorphism for all $n$. We may therefore assume that $A$ and $B$ are artinian. 
Since the map $\psi$ is an isomorphism modulo $\mathfrak{m}_{A}$, it is enough to show that $B$ is flat over $A$ (by Nakayama's lemma). The result therefore follows from the local criterion for flatness ([1], page 91$)$.

To prove that $\psi$ is an isomorphism, it therefore suffices to show that for every integer $k$ the dimensions of $\mathfrak{m}_{R}^{k} / \mathfrak{m}_{R}^{k+1}$ and $\mathfrak{m}^{k} / \mathfrak{m}^{k+1}$ are equal. But we can compute these dimensions: if we define $N(k, l)$ to be the number of monomials of degree $k$ in $l$ variables, then the dimensions of $\mathfrak{m}_{R}^{k} / \mathfrak{m}_{R}^{k+1}$ and $\mathfrak{m}^{k} / \mathfrak{m}^{k+1}$ are both equal to

$$
N(k, l)-N(k-h, l)=N(k, n+s)-N(k-h, n+s) .
$$

Hence $\psi$ is an isomorphism.

Next we claim that $f$ cannot be of the form

$$
f(\underline{Y})=Y_{i_{1}} \cdots Y_{i_{h}}-Y_{i_{1}^{\prime}} \cdots Y_{i_{m}^{\prime}} .
$$

Since $P / Q$ is a group, for every $p_{i_{j}} \in P$ there exists an element $p_{-i_{j}}$ such that

$$
\pi\left(p_{i_{j}}\right)+\pi\left(p_{-i_{j}}\right)=0
$$

in $P / Q$. Writing $p_{-i_{j}}=\sum n_{a} p_{i_{a}}$ we conclude that there exists natural numbers $n_{a}$ such that

$$
p_{i_{j}}+\sum_{a=1}^{n} n_{a} p_{i_{a}} \in \pi^{-1}(0) .
$$

By lemma 2.7, $\pi^{-1}(0)=Q$ and hence there exists an element $q \in Q$ such that

$$
p_{i_{j}}+\sum_{a=1}^{n} n_{a} p_{i_{a}}=q .
$$

Since $p_{i_{j}}$ is not a unit, $q$ is non-zero, and hence there exist natural numbers $n_{a}$ such that $Y_{i_{j}} \prod_{a=1}^{n} Y_{i_{a}}^{n_{a}}=0$.

Since $\widehat{\mathcal{O}}_{X, t}$ is reduced, if $\prod_{j} Y_{i_{j}}^{m_{j}}=0$ with the $m_{j} \geq 1$ then $\prod_{j} Y_{i_{j}}=0$. It follows that $\prod_{j} Y_{i_{j}}=0$. This is impossible if

$$
f(\underline{Y})=Y_{i_{1}} \cdots Y_{i_{h}}-Y_{i_{1}^{\prime}} \cdots Y_{i_{m}^{\prime}} .
$$

Therefore we have an isomorphism

$$
k\left[\left[T_{1}, \ldots, T_{s}, Y_{i_{1}}, \ldots, Y_{i_{n}}\right]\right] /\left(Y_{i_{1}} \cdots Y_{i_{h}}\right) \simeq \widehat{\mathcal{O}}_{X, t}
$$

for some $h$.

We claim that $h=n$. To see this note that

$$
\sum_{j=1}^{h} p_{i_{j}}=p+q
$$


for some $p \in P$ and a nonzero $q \in Q$. If $p_{i_{a}}$ is another irreducible element with $a>h$, then since $P / Q$ is a group there exists an element $p_{-i_{a}}$ as in 2.7 such that $p_{i_{a}}+p_{-i_{a}}$ maps to zero in $P / Q$. Write

$$
p_{-i_{a}}=\sum_{j=1}^{n} b_{i_{j}} p_{i_{j}}
$$

Then at least one of the $b_{i_{j}}$ with $1 \leq j \leq h$ must be zero since otherwise we can write

$p_{-i_{a}}=\sum_{j=1}^{h} p_{i_{j}}+\sum_{j=1}^{h}\left(b_{i_{j}}-1\right) p_{i_{j}}+\sum_{j>h} b_{i_{j}} p_{i_{j}}=p+q+\sum_{j>h} b_{i_{j}} p_{i_{j}}+\sum_{j=1}^{h}\left(b_{i_{j}}-1\right) p_{i_{j}}$

contradicting the minimality of $p_{-i_{a}}$. It follows that $\prod_{j} Y_{i_{j}}^{b_{i_{j}}} \notin J$. On the other hand $p_{i_{a}}+p_{-i_{a}} \in \pi^{-1}(0)=Q$ and since $p_{i_{a}}$ is not a unit $p_{i_{a}}+p_{-i_{a}}$ is a non-zero element in $Q$. Therefore $Y_{i_{a}} \prod_{j} Y_{i_{j}}^{b_{i_{j}}} \in J$, and hence $Y_{i_{a}}$ is a zero divisor in the ring

$$
k\left[\left[T_{1}, \ldots, T_{s}, Y_{i_{1}}, \ldots, Y_{i_{n}}\right]\right] /\left(Y_{i_{1}} \cdots Y_{i_{h}}\right) .
$$

This is a contradiction so we must have $h=n$. This completes the proof of part 1 of the lemma.

To see the second part, suppose we have an equality

$$
\sum m_{j} p_{i_{j}}=\sum m_{j}^{\prime} p_{i_{j}}
$$

in $P$. Since $P$ is cancellative, we can assume that at least one $m_{j}=0$ and hence we get

in the ring

$$
\prod Y_{i_{j}}^{m_{j}}=\prod Y_{i_{j}}^{m_{j}^{\prime}} \neq 0
$$

$$
k\left(s_{0}\right)\left[\left[T_{1}, \ldots, T_{s}, Y_{i_{1}}, \ldots, Y_{i_{n}}\right]\right] /\left(\prod Y_{i_{j}}\right) .
$$

It follows that $m_{j}=m_{j}^{\prime}$ for all $j$. This completes the proof of lemma 2.11 .

From lemmas 2.7.3 and 2.11 it follows that the congruence relation on $Q \oplus \mathbb{N}^{n}$ defined by the map to $P$ is generated by equalities

$$
\sum_{j} n_{i_{j}} p_{i_{j}}=q
$$

where all the $n_{i_{j}}$ are greater than 0 . In addition, from 2.11 we see that we must have an equality

$$
\sum_{j=1}^{n} p_{i_{j}}=q_{0}
$$


for some $q_{0} \in Q$. This proves (1) of the proposition. Now suppose we had an equality in $P$

$$
\sum_{j} n_{i_{j}} p_{i_{j}}=q^{\prime}
$$

for some $q^{\prime} \in Q$ and all $n_{i_{j}} \geq 1$. Then after perhaps reordering the $p_{i_{j}}$ we can assume that $n_{i_{1}}$ is the smallest of the $n_{i_{j}}$. This gives

$$
q^{\prime}=\sum_{j} n_{i_{j}} p_{i_{j}}=n_{i_{1}} q_{0}+\sum_{j}\left(n_{i_{j}}-n_{i_{1}}\right) p_{i_{j}} .
$$

The integrality of $Q \rightarrow P$ now implies that $\sum_{j}\left(n_{i_{j}}-n_{i_{1}}\right) p_{i_{j}}$ is in $Q$ which from above is only possible if $n_{i_{j}}=n_{i_{1}}$ for all $j$. This implies (2).

To see (3), note that the map

$$
Q \oplus_{\mathbb{N}} \mathbb{N}^{n} \rightarrow P
$$

surjective by the definition of the $p_{i_{j}}$. To see that it is injective, suppose $\left(q,\left(\underline{n}_{j}\right)\right)$ and $\left(q^{\prime},\left(\underline{m}_{j}\right)\right)$ map to the same element. Without loss of generality, we may assume that at least one $n_{j}=0$ and at least one $m_{j^{\prime}}=0$ (since there exists $q_{0} \in Q$ such that $\left(q_{0}, 0\right)=(0,(1, \ldots, 1))$ in $\left.Q \oplus_{\mathbb{N}} \mathbb{N}^{n}\right)$. Then we get an equality

$$
q+\sum n_{j} p_{i_{j}}=q^{\prime}+\sum m_{j} p_{i_{j}}
$$

in $P$, and by the integrality we get that there exists $p \in P$ and $q_{3}, q_{4} \in Q$ such that

$$
\sum n_{j} p_{i_{j}}=p+q_{3}, \quad \sum m_{j} p_{i_{j}}=p+q_{4}, \quad q_{3}+q=q_{4}+q^{\prime} .
$$

Since $\sum p_{i_{j}} \in Q$ we may assume that $p=\sum_{j} b_{i_{j}} p_{i_{j}}$ with at least one $b_{i_{j}}=0$. If $q_{3}$ is non-zero we get that $\prod Y_{i_{j}}^{n_{j}}=0$ in $k\left[Y_{i_{1}}, \ldots, Y_{i_{n}}\right] / J$ which from above is impossible since at least one $n_{j}$ is zero. Thus $q_{3}=0$ and hence

$$
\sum m_{j} p_{i_{j}}=\sum n_{j} p_{i_{j}}+q_{4} .
$$

By the same reasoning using the fact that at least one $m_{j^{\prime}}=0$ we conclude that $q_{4}=0$ and hence $q=q^{\prime}$ and $\left(\underline{n}_{j}\right)=\left(\underline{m}_{j}\right)$. This completes the proof of proposition 2.9 .

Let $L \subset P$ be the submonoid of 2.11.2, and define $K \subset Q$ to be the submonoid generated by the element $\sum_{j} p_{i_{j}}$. Equivalently

$$
K=L \cap Q \subset P \text {. }
$$

If $t^{\prime} \in \underline{X}$ is another singular point lying over some $\zeta \in \operatorname{Spec}(\mathcal{O})$, then the above shows that the submonoid $L_{t^{\prime}} \subset \overline{\mathcal{M}}_{X, \bar{t}^{\prime}}$ generated by the irreducible elements of $\overline{\mathcal{M}}_{X, \bar{t}^{\prime}}$ not in the image of $\overline{\mathcal{M}}_{S, \bar{\zeta}}$ is isomorphic to $\mathbb{N}^{r}$ for some $r$, and that the intersection $K_{t^{\prime}}=L_{t^{\prime}} \cap \overline{\mathcal{M}}_{S, \bar{\zeta}}$ is a free monoid of rank 1 . To check this one may base change to an algebraic closure $\bar{k}$ of $k(\zeta)$ and hence can assume that there exists a chart $Q \rightarrow \mathcal{M}_{S}$ such that $Q \rightarrow \overline{\mathcal{M}}_{S}$ 
is bijective. If $t^{\prime}$ is a closed point the result now follows from the above discussion. If $t^{\prime}$ is not a closed point, then choose a specialization $t^{\prime \prime}$ of $t^{\prime}$ and note that the stalk at $t^{\prime}$ is obtained by the cospecialization map from $t^{\prime \prime}$ ([9], II.2.3).

Lemma 2.13. Suppose a chart as in 2.2 has been chosen over some étale neighborhood of $t$ which we also denote by $\underline{X}$. Let $L_{t}$ and $K_{t}$ be the submonoids of $P$ and $Q$ respectively given by the above construction. Then for any other singular point $t^{\prime}$ of $\underline{X}$ lying over some $\zeta \in \operatorname{Spec}(\mathcal{O})$, the submonoids $L_{\bar{t}^{\prime}} \subset \overline{\mathcal{M}}_{X, \bar{t}^{\prime}}$ and $K_{\bar{t}} \subset \overline{\mathcal{M}}_{S, \bar{\zeta}}$ are equal to the images of $L_{t}$ and $K_{t}$.

Proof. By proposition 2.9, there is an isomorphism

$$
Q \oplus_{K_{t}} L_{t} \simeq P,
$$

and hence an isomorphism

$$
\overline{\mathcal{M}}_{X, \bar{t}^{\prime}} \simeq \overline{\mathcal{M}}_{S, \bar{\zeta}} \oplus_{K_{t}}\left(L_{t} /\left(p_{i_{j}}=0 \text { if } p_{i_{j}} \text { maps to a unit in } \mathcal{O}_{X, \bar{t}^{\prime}}\right) .\right.
$$

This implies the lemma.

Let $D$ be a connected component of the singular locus of the closed fiber. For any point $t \in D$ we get from the above construction a free monoid $K_{t} \subset$ $\overline{\mathcal{M}}_{S, s_{0}}$ of rank 1. Proposition 2.13 implies that this monoid is independent of the choice of $t$. To see this suppose $t^{\prime} \in D$ is a second singular point. Choose étale covers $\left\{U_{i}\right\}$ of $D$ and singular points $t_{i}$ in the image of $U_{i}$ such that over each $U_{i}$ there exists a chart as in 2.13 using $t_{i}$. We can also assume that $t_{1}=t$ and $t_{r}=t^{\prime}$ for some $r$. Since $D$ is connected, there exists a sequence $U_{1}, \ldots, U_{r}$ such that for each $i$ there exists a singular point $x_{i}$ of $U_{i} \times_{X} U_{i+1}$ mapping to $D$. It follows from 2.13 that the submonoid $K_{t_{i}} \subset \overline{\mathcal{M}}_{S}$ defined by $t_{i}$ is equal to the submonoid defined by $x_{i}$ and $x_{i-1}$. We conclude that $K_{t}=K_{t^{\prime}}$.

Lemma 2.14. There exists a unique fine sub-log structure $\tilde{K}_{D} \subset \mathcal{M}_{S}$ whose image in $\overline{\mathcal{M}}_{S, s_{0}}$ is equal to $K_{t}$ for some $t \in D$ (and hence all). Also, there exists a unique fine sub-log structure $\tilde{L}_{D} \subset \mathcal{M}_{X}$ whose image in $\overline{\mathcal{M}}_{X, t^{\prime}}\left(t^{\prime}\right.$ any point of $\underline{X}$ ) is equal to the image of $\tilde{K}_{D}$ if $t^{\prime}$ is not singular or if $t^{\prime}$ does not specialize to a point of $D$ and is equal to $L_{t^{\prime}}$ (notation as in 2.13) otherwise.

Proof. $\tilde{K}_{D}$ and $\tilde{L}_{D}$ are uniquely determined if they exist since we have specified their values on stalks, and $\tilde{K}_{D}$ exists since $\underline{S}$ is the spectrum of a strictly henselian local ring.

To show that $\tilde{L}_{D}$ exists, we first claim that the set of singular points of $\underline{X}$ which specialize to $D$ is a connected component of the singular locus of $\underline{X}$. To see this, let $\underline{X}^{\text {sing }}$ be the closed subset of singular points of $\underline{X}$ with the reduced subscheme structure. Then $\underline{X}^{\text {sing }}$ is a proper scheme over $\operatorname{Spec}(\mathcal{O})$, 
and hence by ([2], 3.1) there is an equivalence of categories between the category of finite étale schemes over $\underline{X}^{\text {sing }}$ and the category of finite étale schemes over $\underline{X}_{0}^{\text {sing }}$ (the reduction of $\underline{X}^{\text {sing }}$ ). In a neighborhood of any point of the closed fiber, $\underline{X}$ is isomorphic to

$$
\operatorname{Spec}\left(\mathcal{O}\left[X_{1}, \ldots, X_{d}\right] /\left(X_{1} \cdots X_{r}-t\right)\right)
$$

for some $d, r \in \mathbb{Z}$ and $t \in \mathcal{O}$, and hence $\underline{X}_{0}^{\text {sing }}$ is equal to the singular locus of the closed fiber $\underline{X}_{0}$ with the reduced structure. Now by the above equivalence, the connected component $D \subset \underline{X}_{0}^{\text {sing }}$ lifts to a finite étale scheme $\tilde{D} \rightarrow \underline{X}^{\text {sing }}$. Since the reduction of this map is of degree 1 , this is in fact a closed immersion, and since it is also étale the scheme $\tilde{D}$ is a connected component of $\underline{X}^{\text {sing }}$ ( $\tilde{D}$ is connected by [2], 3.3). Moreover, the points of $\tilde{D}$ are precisely the singular points which specialize to points of $D$.

It follows that if we choose a chart as in 2.2 in some étale neighborhood of a point $t$ of $D$, then the $\log$ structure defined by $L_{t}$ (see 2.13) has the properties required of $\tilde{L}_{D}$. Moreover, these locally constructed log structures will glue since they are sub-log structures of $\mathcal{M}_{X}$.

Define $\mathcal{M}_{S}^{\sharp}$ to be the amalgemation over all $D$

$$
\oplus \mathcal{O}_{S}^{*} \tilde{K}_{D}
$$

and similarly define $\mathcal{M}_{X}^{\sharp}$ to be

$$
\oplus_{\mathcal{O}_{X}^{*}} \tilde{L}_{D}
$$

Then the natural diagram

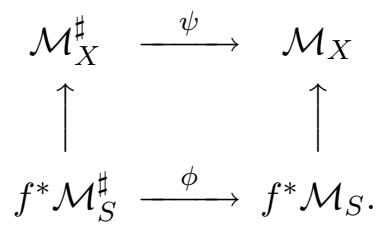

is co-cartesian. By construction $f^{\sharp}$ is smooth, essentially semi-stable, and the pullback to the closed fiber is special .

Lemma 2.15. Suppose $\left(\mathcal{M}_{S}^{\prime}, \mathcal{M}_{X}^{\prime}, \phi^{\prime}, \psi^{\prime}\right)$ is a second collection of data for which

$$
f^{\prime}:\left(\underline{X}, \mathcal{M}_{X}^{\prime}\right) \longrightarrow\left(\underline{S}, \mathcal{M}_{S}^{\prime}\right)
$$

is essentially semi-stable and special at $s_{0}$, and for which the diagram

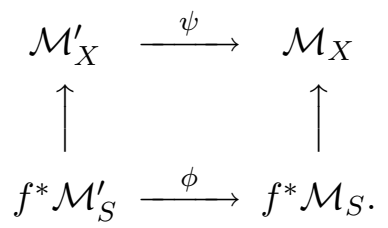


is co-cartesian. Then there exists a unique pair of isomorphisms $\epsilon: \mathcal{M}_{S}^{\sharp} \simeq$ $\mathcal{M}_{S}^{\prime}$ and $\eta: \mathcal{M}_{X}^{\sharp} \simeq \mathcal{M}_{X}^{\prime}$ such that the diagram

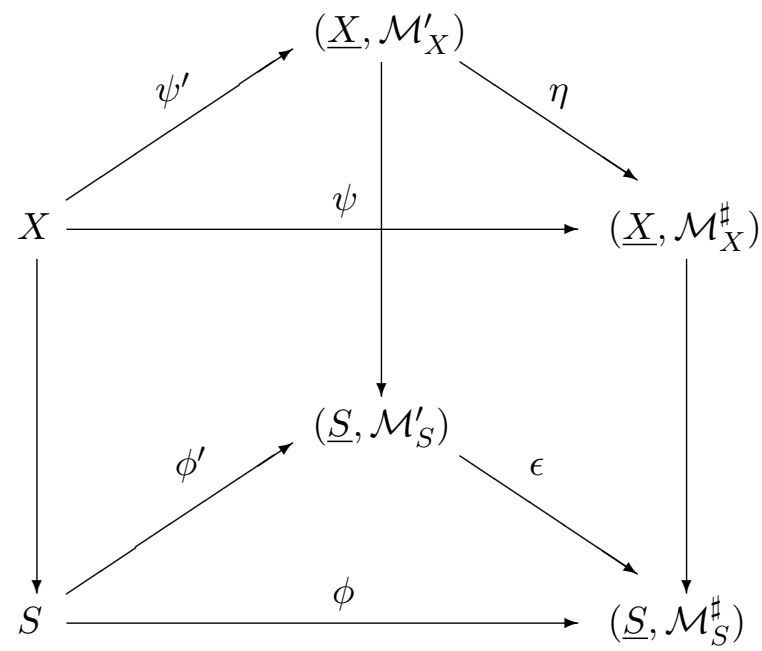

commutes.

Proof. If we apply the above construction to $\left(\underline{X}, \mathcal{M}_{X}^{\prime}\right) /\left(\underline{S}, \mathcal{M}_{S}^{\prime}\right)$, then it follows from chasing through the construction that we recover the data $\left(\mathcal{M}_{S}^{\prime}, \mathcal{M}_{X}^{\prime}, \phi^{\prime}, \psi^{\prime}\right)$. Hence $\mathcal{M}_{S}^{\prime}$ and $\mathcal{M}_{X}^{\prime}$ have canonical decompositions

$$
\mathcal{M}_{S}^{\prime}=\oplus_{\mathcal{O}_{S}^{*}} \tilde{K}_{D}^{\prime}, \quad \mathcal{M}_{X}^{\prime}=\oplus_{\mathcal{O}_{S}^{*}} \tilde{L}_{D}^{\prime} .
$$

In addition the images of $\tilde{L}_{D}^{\prime}$ and $\tilde{K}_{D}^{\prime}$ in $\mathcal{M}_{X}$ and $\mathcal{M}_{S}$ are the sub-log structures obtained by applying the construction to $X / S$. From this the existence of $(\epsilon, \eta)$ follows. The uniqueness follows from the fact that any pair of isomorphisms $(\epsilon, \eta)$ as in the lemma must preserve the decompositions together with the fact that the $\tilde{K}_{D}$ and $\tilde{L}_{D}$ are sub-log structures of $\mathcal{M}_{S}$ and $\mathcal{M}_{X}$ respectively.

To complete the proof of proposition 2.16 in the case when $S$ is the spectrum of a strictly henselian local ring $\mathcal{O}$, and $\mathcal{M}_{S}$ admits a chart $Q \rightarrow \mathcal{M}_{S}$ such that $Q \rightarrow \overline{\mathcal{M}}_{S, s_{0}}$ is bijective, it suffices to show that the morphism

$$
\left(\underline{X}^{\sharp}, \mathcal{M}_{X}^{\sharp}\right) \longrightarrow\left(\underline{S}^{\sharp}, \mathcal{M}_{S}^{\sharp}\right)
$$

constructed above is special at all points of $\underline{S}$ and not just at $s_{0}$. For later use, we prove a slightly stronger statement:

Proposition 2.16. Let $f: X \rightarrow S$ be as in 2.6 (we no longer assume that $S$ is the spectrum of a strictly henselian local ring), and suppose in addition that $f$ is essentially semi-stable. Then the set

$$
V:=\{s \in \underline{S} \mid f \text { is special at } \bar{s}\}
$$


is open.

Proof. By ([1], V.4.4) it suffices to show that $V$ is a constructible set and that if $s \in V$ then every generization of $s$ is also in $V$.

Lemma 2.17. $V$ is constructible.

Proof. The condition is local on $S$ so we may assume that $S=\operatorname{Spec}(A)$ for some ring $A$ and that $\mathcal{M}_{S}$ is induced from some map

$$
\mathbb{N}^{r} \rightarrow A \text {. }
$$

Let $t_{1}, \ldots, t_{r} \in A$ be the images of the standard generators. For each integer $n$ and for each choice of $n$ distinct elements $i_{1}, \ldots, i_{n} \in\{1, \ldots, r\}$ let $A_{n, i}$ be the ring

$$
A_{t_{i_{1} \cdots t_{i_{n}}}} /\left(t_{j}\right)_{j \notin\left\{i_{1}, \ldots, i_{n}\right\}} .
$$

Let $S_{n, i}=\operatorname{Spec}\left(A_{n, i}\right)$. Then each $S_{n, i}$ is a constructible subscheme of $S$, and the union of the $S_{n, i}$ is all of $S$. To prove the lemma it therefore suffices to consider the case of the $S_{n, i}$. This reduces us to the case when the log structure on $S$ is given by a map

$$
\mathbb{N}^{r} \rightarrow A
$$

sending all elements to zero. In addition, by base changing to the irreducible components with the reduced structure we may assume that $A$ is an integral domain. In this case, $\underline{X}$ is étale locally isomorphic to

$$
A\left[X_{1}, \ldots, X_{l}\right] / X_{1} \cdots X_{s}
$$

for some $l$ and $s$. Let $\underline{X}^{\text {sing }}$ be the singular locus of $X$ with the reduced induced structure. From the local description of $\underline{X}$ we see that the geometric fibers of $\underline{X}^{\text {sing }}$ are all reduced and that $\underline{X}^{\text {sing }}$ is flat over $A$. It follows that the function

$$
s \mapsto \text { number of connected components of } \underline{X}_{\bar{s}}^{\text {sing }}
$$

can be identified with the function

$$
s \mapsto h^{0}\left(\underline{X}^{\text {sing }} \times_{S} \operatorname{Spec}(k(s)), \mathcal{O}_{\underline{X}^{\operatorname{sing}} \times_{S} \operatorname{Spec}(k(s))}\right) .
$$

By the semi-continuity theorem ([8], III.12.8), we conclude that the function

$$
s \mapsto \text { number of connected components of } \underline{X}_{\bar{s}}^{\text {sing }}
$$

is upper semi-continous. It follows that the set of points $s$ where this number is equal to $r$ is constructible. On the other hand, the set of points $s$ where the map in definition 2.5 is surjective is open by the description of the charts in lemma 2.2. Therefore the set of points where the map in 2.5 is bijective is constructible.

Lemma 2.18. $V$ is stable under generization. 
Proof. We can assume that $S=\operatorname{Spec}(A)$, where $A$ is a local ring and that we want to prove that if the closed fiber is special then the fiber over some prime $\mathfrak{p} \subset A$ is also. Replacing $A$ by $A / \mathfrak{p}$ we may assume that $A$ is a local integral domain and that we wish to prove that the generic fiber is special if the closed fiber is special. In addition by replacing $A$ by an étale cover we may assume that we have a global chart

$$
\mathbb{N}^{T} \rightarrow \mathcal{M}_{S}
$$

where $T$ is the set of connected components of the singular locus of $\underline{X}_{\bar{s}_{0}}$. Let $n_{t} \in \mathbb{N}^{T}$ be an irreducible element such that $\alpha\left(n_{t}\right)=0$, and let $\underline{Z}$ denote the set of singular points of $\underline{X}$ which map to $n_{t}$ under the map in definition 2.5. $\underline{Z}$ is a closed set, and we view $\underline{Z}$ as a subscheme with the reduced structure. What has to be shown is that the generic fiber of $\underline{Z}$ is non-empty and geometrically connected.

Let $t_{0}$ be a point of the closed fiber whose corresponding element of $\mathbb{N}^{T}$ is $n_{t}$. In an étale neighborhood of $t_{0}$ there exists an étale morphism

$$
\underline{U} \rightarrow \operatorname{Spec}\left(A\left[X_{1}, \ldots, X_{n}\right] / X_{1} \cdots X_{s}\right)
$$

for some $n$ and $s$. From this it follows that the generic fiber of $\underline{Z}$ is not empty. From this local description it also follows that for each point $s \in \underline{S}$ the fiber $\underline{Z}_{s}$ is reduced and that $\underline{Z}$ is flat over $A$. Therefore, the generic fiber of $\underline{Z}$ is geometrically connected if and only if

$$
h^{0}\left(\underline{Z} \otimes_{A} \operatorname{Frac}(A), \mathcal{O}_{\underline{Z} \otimes_{A} \operatorname{Frac}(A)}\right)=1
$$

where $\operatorname{Frac}(A)$ denotes the field of fractions of $A$. This number is at least 1 since $\underline{Z} \otimes_{A} \operatorname{Frac}(A)$ is not empty. On the other hand, the semi-continuity theorem ([8], III.12.8) combined with the fact that the closed fiber of $\underline{Z}$ is geometrically connected implies that the generic fiber of $\underline{Z}$ is geometrically connected. This implies the lemma.

Proposition 2.16 now follows from the two lemmas.

We can now complete the proof of theorem 2.6. By the uniqueness we may assume that $S$ is affine. Note first that if $s$ is a point and $S_{s}=\operatorname{Spec}\left(\mathcal{O}_{S, \bar{s}}\right)$, then by $([16], 4.3)$ there is an equivalence of categories

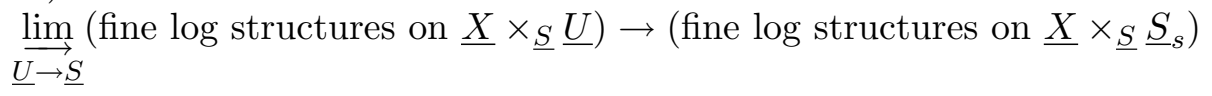

where the limit is taken over étale neighborhoods $\underline{U} \rightarrow \underline{S}$ of $\bar{s}$.

It follows that if we have two collections of data

$$
\left(\mathcal{M}_{S, 1}^{\sharp}, \mathcal{M}_{X, 1}^{\sharp}, \phi_{1}, \psi_{1}\right), \quad\left(\mathcal{M}_{S, 2}^{\sharp}, \mathcal{M}_{X, 2}^{\sharp}, \phi_{2}, \psi_{2}\right)
$$

then there exists exactly one isomorphism between them. Indeed if $s \in S$ is a point then after replacing $S$ by an fppf-cover we can assume that we have a chart $Q \rightarrow \mathcal{M}_{S}$ such that the map $Q \rightarrow \overline{\mathcal{M}}_{S, \bar{s}}$ is bijective. Over the strict 
henselization of the local ring of $s$ there is a unique isomorphism, and this isomorphism can by the equivalence 2.3 be extended to some étale neighborhood of $s$. It follows that fppf-locally there exists a unique isomorphism, and since a faithfully flat morphism of finite presentation is a morphism of effective descent for fine log structures ([16], 2.13) there exists a global isomorphism.

To prove existence, it suffices by $([16], 2.13)$ to prove existence in some fppf-neighborhood of a point $s \in S$. Thus we may assume that there exists a chart $Q \rightarrow \mathcal{M}_{S}$ such that $Q \rightarrow \overline{\mathcal{M}}_{S, \bar{s}}$ is bijective. We have shown that there exists data $\left(\mathcal{M}_{S}^{\sharp}, \mathcal{M}_{X}^{\sharp}, \phi, \psi\right)$ over the strict henselization of the local ring at $s$. From the equivalence 2.3 it follows that we can extend the data $\left(\mathcal{M}_{S}^{\sharp}, \mathcal{M}_{X}^{\sharp}, \phi, \psi\right)$ over the henselization to some étale neighborhood of $s$. After perhaps shrinking $S$ we can assume that the resulting morphism is still $\log$ smooth. We claim that after perhaps shrinking $S$ some more the morphism $f^{\sharp}$ will be essentially semi-stable. For this we may assume that there exists a chart $\mathbb{N}^{T} \rightarrow \mathcal{M}_{S}^{\sharp}$ where $T$ denotes the set of connected component of the singular locus of $\underline{X}_{\bar{s}}$. Consider the set $\underline{V}$ of points $t \in \underline{X}$ for which the map

$$
\left(f^{-1} \overline{\mathcal{M}}_{S}^{\sharp}\right)_{\bar{t}} \rightarrow \overline{\mathcal{M}}_{X, \bar{t}}^{\sharp}
$$

is as in the definition of essentially semi-stable. By lemma 2.2, the set $\underline{V}$ is open and by construction $\underline{V}$ contains every point of the fiber over $s$. Since $f$ is proper the image of $\underline{V}^{c}$ in $\underline{S}$ is a closed set not containing $s$. Therefore by replacing $S$ by the complement of $f\left(\underline{V}^{c}\right)$ we may assume that $f^{\sharp}$ is essentially semi-stable everywhere.

It was also shown above that the set of points where the morphism $f^{\sharp}$ is special is open on the base, and hence after shrinking $S$ some more we have constructed datum $\left(\mathcal{M}_{S}^{\sharp}, \mathcal{M}_{X}^{\sharp}, \phi, \psi\right)$ satisfying the conditions of 2.6 in an fppf-neighborhood of $s$. This concludes the proof of theorem 2.6.

\section{Log StRuctures of EMBEDding AND SEMI-STABLE TYPE}

Fix a scheme $\underline{S}$ and a global section $t \in \Gamma\left(\underline{S}, \mathcal{O}_{\underline{S}}\right)$. Let $\mathcal{M}_{S}$ denote the $\log$ structure on $\underline{S}$ associated to the map $\mathbb{N} \rightarrow \mathcal{O}_{S}$ sending 1 to $t$, and let $S$ be the $\log$ scheme $\left(\underline{S}, \mathcal{M}_{S}\right)$. We propose the following definition as a generalization of F. Kato's notion of a log structure of semi-stable type:

Definition 3.1. A log smooth morphism $f: X \rightarrow S$ is $t$-semi-stable if for every geometric point $\bar{x} \rightarrow \underline{X}$ the stalk $\overline{\mathcal{M}}_{X, \bar{x}}$ is a free monoid and the map

$$
\mathbb{N} \longrightarrow \overline{\mathcal{M}}_{S, f(\bar{x})} \longrightarrow \overline{\mathcal{M}}_{X, \bar{x}}
$$

is the diagonal map. If the morphism $f: \underline{X} \rightarrow \underline{S}$ is fixed, then we will also refer to a morphism of log structures $f^{*} \mathcal{M}_{S} \rightarrow \mathcal{M}_{X}$ making $\left(\underline{X}, \mathcal{M}_{X}\right) \rightarrow S$ $t$-semi-stable as a $t$-semi-stable log structure on $\underline{X}$. 
Remark 3.2. For any integer $r>0$, the diagonal map $\mathbb{N} \rightarrow \mathbb{N}^{r}$ is invariant under all automorphisms of $\mathbb{N}^{r}$, and hence for any free monoid $M$ there is a canonical diagonal map $\mathbb{N} \rightarrow M$. This is the map referred to in the definition.

We will also study a generalization of F. Kato's notion of a log structure of embedding type:

Definition 3.3. Let $f: \underline{X} \rightarrow \underline{S}$ be a morphism of schemes. A log structure $\mathcal{M}_{X}$ on $\underline{X}$ is of embedding type relative to $t$ if the morphism $\left(\underline{X}, \mathcal{M}_{X}\right) \rightarrow\left(\underline{S}, \mathcal{O}_{S}^{*}\right)$ factors étale locally through a $t$-semi-stable morphism $\left(\underline{X}, \mathcal{M}_{X}\right) \rightarrow S$. If $f: X \rightarrow\left(\underline{S}, \mathcal{O}_{S}^{*}\right)$ is a morphism of log schemes, then we also say that $f$ is of embedding type relative to $t$ if $\mathcal{M}_{X}$ is a log structure of embedding type relative to $t$ on $\underline{X}$.

It follows from ([15], 2.25) that if $f: X \rightarrow S$ is $t$-semi-stable (resp. $f: X \rightarrow\left(\underline{S}, \mathcal{O}_{S}^{*}\right)$ is of embedding type relative to $\left.t\right)$, then in an étale neighborhood of any closed point the $\log$ structure $\mathcal{M}_{X}$ admits a chart of the following form:

Definition 3.4. Suppose $\mathcal{M}_{X}$ is a $t$-semi-stable log structure (resp. a log structure of embedding type relative to $t$ ) on $\underline{X}$. A standard chart for $\mathcal{M}_{X}$ at a closed point $x \in \underline{X}$ is a strict, étale morphism of log schemes over $S\left(\operatorname{resp}\right.$. over $\left.\left(\underline{S}, \mathcal{O}_{S}^{*}\right)\right)$

$$
\left(\underline{X}, \mathcal{M}_{X}\right) \longrightarrow\left(\operatorname{Spec}\left(\mathcal{O}_{S}\left[X_{1}, \ldots, X_{l}\right] /\left(X_{1} \cdots X_{r}-t\right)\right) \text {, standard log str. }\right)
$$

for some $l$ and $r$, which sends $x$ to the point $X_{1}=\cdots=X_{l}=0$ if $r>1$, and sends $x$ to the point $X_{1}=t, X_{2}=\cdots=X_{l}=0$ if $r=1$.

Remark 3.5. The standard log structure referred to in the definition is that induced by the map

$$
\mathbb{N}^{r} \longrightarrow \mathcal{O}_{S}\left[X_{1}, \ldots, X_{l}\right] /\left(X_{1} \cdots X_{r}-t\right), \quad\left(n_{j}\right)_{j=1}^{r} \mapsto \prod_{j=1}^{r} \underline{X}_{j}^{n_{j}}
$$

The diagonal map $\mathbb{N} \rightarrow \mathbb{N}^{r}$ naturally gives

$$
\left(\operatorname{Spec}\left(\mathcal{O}_{S}\left[X_{1}, \ldots, X_{l}\right] /\left(X_{1} \cdots X_{r}-t\right)\right), \text { standard log str. }\right)
$$

the structure of a log scheme over $S$.

For the remainder of this section we will fix a morphism of schemes $f$ : $\underline{X} \rightarrow \underline{S}$ such that étale locally on $\underline{X}$ there exists a log structure $\mathcal{M}_{X}$ of embedding type relative to $t$.

Proposition 3.6. Any two log structures on $\underline{X}$ of embedding type relative to $t$ are locally isomorphic. Moreover, if $\phi: \mathcal{M}_{X} \rightarrow \mathcal{M}_{X}$ is an automorphism of a log structure of embedding type relative to $t$, then the induced map $\bar{\phi}: \overline{\mathcal{M}}_{X} \rightarrow \overline{\mathcal{M}}_{X}$ is the identity. 
Proof. Let $\mathcal{M}_{1}$ and $\mathcal{M}_{2}$ be two log structures of embedding type relative to $t$, and let $x \in \underline{X}$ be a closed point. We construct an isomorphism $\mathcal{M}_{1} \simeq \mathcal{M}_{2}$ in some étale neighborhood of $x$.

To do this, we may assume that we have standard charts

$$
\begin{gathered}
\pi_{1}: \underline{X} \longrightarrow \operatorname{Spec}\left(\mathcal{O}_{S}\left[X_{1}, \ldots, X_{d}\right] /\left(X_{1} \cdots X_{r}-t\right)\right) \\
\pi_{2}: \underline{X} \longrightarrow \operatorname{Spec}\left(\mathcal{O}_{S}\left[Y_{1}, \ldots, Y_{d^{\prime}}\right] /\left(Y_{1} \cdots Y_{r^{\prime}}-t\right)\right)
\end{gathered}
$$

sending $x$ to the points $X_{1}=\cdots=X_{d}=0$ and $Y_{1}=\cdots=Y_{d^{\prime}}=0$. We show that $d=d^{\prime}, r=r^{\prime}$, and that there exists a unique automorphism $\sigma$ of the set $\{1, \ldots, r\}$ such that in some étale neighborhood of $x$ there exists solutions to the equations

$$
\pi_{1}^{*}\left(X_{i}\right)=U_{i} \pi_{2}^{*} Y_{\sigma(i)}, \quad U_{i} V_{i}=1, \quad i=1, \ldots, r .
$$

By the Artin approximation theorem ([2], 1.10), it suffices to show that there exists solutions in the completion $\widehat{\mathcal{O}}_{X, x}$. Let $\mathfrak{m}$ denote the maximal ideal of $\widehat{\mathcal{O}}_{X, x}, \mathfrak{m}_{\widehat{\mathcal{O}}_{S, f(x)}}$ the ideal in $\widehat{\mathcal{O}}_{X, x}$ generated by the maximal ideal of $\widehat{\mathcal{O}}_{S, f(x)}$, and let

$\phi: \widehat{\mathcal{O}}_{S, f(x)}\left[\left[X_{1}, \ldots, X_{d}\right]\right] /\left(X_{1} \cdots X_{r}-t\right) \simeq \widehat{\mathcal{O}}_{S, f(x)}\left[\left[Y_{1}, \ldots, Y_{d^{\prime}}\right]\right] /\left(Y_{1} \cdots Y_{r^{\prime}}-t\right)$ be the isomorphism induced by $\pi_{1}$ and $\pi_{2}$. By looking at the dimension of $\widehat{\mathcal{O}}_{X, x} /\left(\mathfrak{m}_{\widehat{\mathcal{O}}_{S, f(x)}}, \mathfrak{m}^{2}\right)$ we see that $d=d^{\prime}$.

Consider first the case when $t=0$. In this case, the ring

$$
\widehat{\mathcal{O}}_{S, f(x)}\left[\left[Y_{1}, \ldots, Y_{d^{\prime}}\right]\right] /\left(Y_{1} \cdots Y_{r^{\prime}}\right)
$$

is naturally graded by degree in the $Y_{i}$. For $i=1, \ldots, r$ write

$$
\phi\left(X_{i}\right)=L_{i}(\underline{Y})+H_{i}(\underline{Y})
$$

where $L_{i}(\underline{Y})$ consists of linear terms and $H_{i}(\underline{Y})$ consists of terms of degree 2 or greater.

Lemma 3.7. 1. For any ring $\Lambda$ and any $i \in\{1, \ldots, r\}$, the kernel of multiplication by $Y_{1} \cdots \widehat{Y}_{i} \cdots Y_{r}$ on

$$
\Lambda\left[Y_{1}, \ldots, Y_{d}\right] /\left(Y_{1} \cdots Y_{r}\right) \quad\left(\text { resp. } \Lambda\left[\left[Y_{1}, \ldots, Y_{d}\right]\right] /\left(Y_{1} \cdots Y_{r}\right)\right)
$$

is equal to the ideal generated by $Y_{i}$.

2. There exists a unique permutation $\sigma$ of $\{1, \ldots, r\}$ such that for each $i \in$ $\{1, \ldots, r\}$ there exists a unit $u_{i} \in \widehat{\mathcal{O}}_{S, f(x)}^{*}$ such that $L_{i}(\underline{Y})=u_{i} Y_{\sigma(i)}$.

Proof. 1 . As a module over $\Lambda$, the ring $\Lambda\left[Y_{1}, \ldots, Y_{d}\right] /\left(Y_{1} \cdots Y_{r}\right)$ is isomorphic to the direct sum

$$
\oplus_{\underline{l} \in I} \Lambda \underline{\underline{l}}
$$

where $I \subset \mathbb{N}^{d}$ is the subset of $d$-tuples $\left(l_{1}, \ldots, l_{d}\right)$ with at least one $l_{i}=0$ for $1 \leq i \leq r$. Part 1 follows from the fact that multiplication by $Y_{1} \cdots \widehat{Y}_{i} \cdots Y_{r}$ 
preserves this decomposition. Moreover, the case of $\Lambda\left[\left[Y_{1}, \ldots, Y_{d}\right]\right] /\left(Y_{1} \cdots Y_{r}\right)$ also follows since this ring is flat over $\Lambda\left[Y_{1}, \ldots, Y_{d}\right] /\left(Y_{1} \cdots Y_{r}\right)$.

2. Let $\mathfrak{n}$ be the maximal ideal of $\widehat{\mathcal{O}}_{S, f(x)}$. We first reduce to the case when $\mathfrak{n}=0$. Evidently it suffices to prove the uniqueness of $\sigma$ in this special case. Now suppose we have found units $u_{i} \in\left(\mathcal{O}_{S, f(x)} / \mathfrak{n}^{n}\right)^{*}$ such that

$$
L_{i}(\underline{Y}) \equiv u_{i} Y_{\sigma(i)} \bmod \mathfrak{n}^{n} .
$$

We construct units $u_{i}^{\prime} \in\left(\mathcal{O}_{S, f(x)} / \mathfrak{n}^{n+1}\right)^{*}$ such that

$$
L_{i}(\underline{Y}) \equiv u_{i}^{\prime} Y_{\sigma(i)} \quad \bmod \mathfrak{n}^{n+1}
$$

and such that there exists an integer $n_{0}$ which is independent of $n$ for which

$$
u_{i} \equiv u_{i}^{\prime} \bmod \mathfrak{n}^{n-n_{0}} .
$$

To do this, choose a lifting $\tilde{u}_{i}$ of $u_{i}$ to $\widehat{\mathcal{O}}_{S, f(x)}^{*}$, and let $D_{i}(\underline{Y})=L_{i}(\underline{Y})-$ $\tilde{u}_{i} Y_{\sigma(i)}$. Then we get

$$
0 \equiv \sum_{i=1}^{r} \tilde{u}_{1} \cdots \widehat{\tilde{u}}_{i} \cdots \tilde{u}_{r} D_{i}(\underline{Y}) Y_{1} \cdots \widehat{Y}_{\sigma(i)} \cdots Y_{r} \quad \bmod \left(\mathfrak{n}^{n+1},\left(Y_{1} \cdots, Y_{r}\right)^{r+1}\right) .
$$

Since the $D_{i}(\underline{Y})$ are linear, this implies that

$$
D_{i}(\underline{Y}) \equiv Y_{\sigma(i)} F_{i} \quad \bmod \mathfrak{n}^{n+1}
$$

for some $F_{i} \in \mathcal{O}_{S, f(x)}$. By the Artin-Rees lemma, there exists an integer $n_{0}$, which is independent of $n$, such that

$$
\left(Y_{\sigma(i)}\right) \cap \mathfrak{n}^{n} \cdot \widehat{\mathcal{O}}_{X, x} \subset \mathfrak{n}^{n-n_{0}} \cdot\left(Y_{\sigma(i)}\right),
$$

and hence we may choose $F_{i}$ to be in $\mathfrak{n}^{n-n_{0}}$. We define $u_{i}^{\prime}$ to be $\tilde{u}_{i}+F_{i}$. This proves that it suffices to consider the case when $\mathfrak{n}=0$.

Assuming now that $\mathfrak{n}=0$, observe that the $X_{i}$ map to non-zero elements in $\mathfrak{m} / \mathfrak{m}^{2}$, and hence the terms $L_{i}(\underline{Y})$ are non-zero. Since $t=0$ we find that

$$
0=\prod_{i=1}^{r} \phi\left(X_{i}\right)=\prod_{i=1}^{r} L_{i}(\underline{Y})+\text { terms of degree } \geq r+1 .
$$

From this we conclude that $r \geq r^{\prime}$ and hence by symmetry $r=r^{\prime}$. Moreover, by looking at degrees in the $Y_{i}$, one finds that $L_{i}(\underline{Y})=u_{i} Y_{\sigma(i)}$ for a unique permutation $\sigma$ of $\{1, \ldots, r\}$ and some unit $u_{i} \in \widehat{\mathcal{O}}_{S, f(x)}^{*}$.

Remark 3.8. Part 1 of the lemma implies that the kernel of multiplication by $Y_{1} \cdots \widehat{Y}_{i} \cdots Y_{r}$ on $\mathcal{O}_{S}\left[Y_{1}, \ldots, Y_{d}\right] /\left(Y_{1} \cdots Y_{r}-t\right)$ is equal to the ideal $K_{t} \cdot\left(Y_{i}\right)$, where $K_{t}$ is the kernel of multiplication by $t$ on $\mathcal{O}_{S}$. Indeed the case $t=0$ implies that any element killed by $Y_{1} \cdots \widehat{Y}_{i} \cdots Y_{r}$ can be written as

$$
f_{1} Y_{i}+f_{2} t=\left(f_{1}+f_{2} Y_{1} \cdots \widehat{Y}_{i} \cdots Y_{r}\right) Y_{i}
$$

where $t\left(f_{1}+f_{2} Y_{1} \cdots \widehat{Y}_{i} \cdots Y_{r}\right)=0$. Since $\mathcal{O}_{S}\left[Y_{1}, \ldots, Y_{d}\right] /\left(Y_{1} \cdots Y_{r}-t\right)$ is flat over $\mathcal{O}_{S}$, this implies that $\left(f_{1}+f_{2} Y_{1} \cdots \widehat{Y}_{i} \cdots Y_{r}\right)$ is in $K_{t} \cdot \mathcal{O}_{X}$. 
Next we show that every term in $H_{i}(\underline{Y})$ can be written as a multiple of $Y_{\sigma(i)}$. We proceed by induction on the degree of monomials. Suppose true for monomials of degree smaller than $n$ in $H_{i}(\underline{Y})$. Then we can find a unit $u_{i}$ which is a polynomial of degree less than or equal to $n-2$ such that

$$
\phi\left(X_{i}\right) \equiv u_{i} Y_{\sigma(i)} \bmod \left(Y_{1}, \ldots, Y_{d^{\prime}}\right)^{n} .
$$

By looking at monomial terms of degree $r-1+n$ in $\prod_{i=1}^{r} \phi\left(X_{i}\right)$ we find that $Y_{1} \cdots \hat{Y}_{\sigma(i)} \cdots Y_{r}$ kills the degree $n$ part of $H_{i}(\underline{Y})$. From this it follows that the degree $n$ part of $H_{i}(\underline{Y})$ can be written as a multiple of $Y_{\sigma(i)}$. This completes the induction step and the proof of the case when $t=0$.

Next suppose $t \neq 0$. By the Artin-Rees lemma there exists an integer $m_{0}$ such that for all $n$

$$
\left(Y_{\sigma(i)}\right) \cap \mathfrak{m}_{\widehat{\mathcal{O}}_{S, f(x)}}^{n} \subset \mathfrak{m}_{\widehat{\mathcal{O}}_{S, f(x)}}^{n-m_{0}} \cdot\left(Y_{\sigma(i)}\right) .
$$

Given an integer $n$ and units $u_{i}$ such that

$$
\phi\left(X_{i}\right) \equiv u_{i} Y_{\sigma(i)} \quad \bmod \mathfrak{m}_{\widehat{\mathcal{O}}_{S, f(x)}}^{n}
$$

we construct units $u_{i}^{\prime}$ such that

and such that

$$
\phi\left(X_{i}\right) \equiv u_{i}^{\prime} Y_{\sigma(i)} \quad \bmod \mathfrak{m}_{\widehat{\mathcal{O}}_{S, f(x)}^{n+1}}
$$

$$
u_{i}^{\prime} \equiv u_{i} \quad \bmod \mathfrak{m}_{\widehat{\mathcal{O}}_{S, f(x)}^{n-m_{0}}}^{n}
$$

Let $H_{i}(\underline{Y})=\phi\left(X_{i}\right)-u_{i} Y_{\sigma(i)}$. Then

$$
t \equiv \prod_{i=1}^{r} u_{i} Y_{\sigma(i)}+\sum_{i=1}^{r} u_{1} \cdots \hat{u}_{i} \cdots u_{r} Y_{1} \cdots \hat{Y}_{\sigma(i)} \cdots Y_{r} H_{i}(\underline{Y}) \quad \bmod \mathfrak{m}_{\widehat{\mathcal{O}}_{S, f(x)}^{n+1}}^{n}
$$

and hence

$$
Y_{1} \cdots \hat{Y}_{\sigma(i)} \cdots Y_{r} H_{i}(\underline{Y}) \equiv 0 \quad \bmod \left(t, \mathfrak{m}_{\left.\widehat{\mathcal{O}}_{S, f(x)}^{n+1}\right)}\right) .
$$

Since $t \in\left(Y_{\sigma(i)}\right)$, it follows that

$$
H_{i}(\underline{Y}) \equiv 0 \quad \bmod \left(\left(Y_{\sigma(i)}\right) \cap \mathfrak{m}_{\widehat{\mathcal{O}}_{S, f(x)}}^{n}, \mathfrak{m}_{\widehat{\mathcal{O}}_{S, f(x)}^{n+1}}^{n+1} .\right.
$$

Hence by definition of $m_{0}$, there exists $F_{i} \in \mathfrak{m}_{\widehat{\mathcal{O}}_{S, f(x)}}^{n-m_{0}}$ such that

$$
H_{i}(\underline{Y}) \equiv Y_{\sigma(i)} F_{i} \quad \bmod \mathfrak{m}_{\widehat{\mathcal{O}}_{S, f(x)}^{n+1}} .
$$

This completes the proof of the existence of solutions to equations 3.1 in the completion $\widehat{\mathcal{O}}_{X, x}$ and hence the proof of existence of an isomorphism between $\mathcal{M}_{1}$ and $\mathcal{M}_{2}$ in an étale neighborhood of $x$. The second statement in the proposition follows from the uniqueness of $\sigma$.

The proposition and its proof implies that there are various canonically defined sheaves on $\underline{X}$ : 
Construction 3.9. The uniqueness of $\bar{\sigma}$ in the proposition implies that for any $\log$ structure $\mathcal{M}_{X}$ of embedding type relative to $t$, the sheaf $\overline{\mathcal{M}}_{X}$ is invariant under all automorphisms. Hence there is a canonically defined sheaf $\overline{\mathcal{M}}$ on all of $\underline{X}$ since there exists a log structure of embedding type on $\underline{X}$ étale locally.

Construction 3.10. If

$$
\pi: \underline{X} \rightarrow \operatorname{Spec}\left(\mathcal{O}_{S}\left[X_{1}, \ldots, X_{d}\right] /\left(X_{1} \cdots X_{r}-t\right)\right)
$$

is a standard chart for a log structure of embedding type relative to $t$ on $\underline{X}$, then the ideal

$$
J:=\left(\ldots, X_{1} \cdots \widehat{X}_{i} \cdots X_{r}, \ldots\right)_{i=1}^{r}
$$

is independent of the choice of chart, and hence there exists a globally defined ideal sheaf $J \subset \mathcal{O}_{X}$. We let $\underline{D} \subset \underline{X}$ be the resulting closed subscheme.

Construction 3.11. If $\phi$ is a chart as in 3.4, let $G \subset \mathcal{O}_{X}^{*}$ be the subsheaf of units $u$ which locally can be written as

$$
u=1+\sum_{i=1}^{r} a_{i} X_{1} \cdots \widehat{X}_{i} \cdots X_{r}
$$

where $t a_{i}=0$. Then $G$ is independent of the choices and has a natural structure of a group: if we define $\underline{Z} \subset \underline{X}$ to be the closed subscheme defined by $J$ times the kernel of multiplication by $t$ on $\mathcal{O}_{X}$, then $G$ is the kernel of the $\operatorname{map} \mathcal{O}_{X}^{*} \rightarrow \mathcal{O}_{Z}^{*}$.

The following is the main result in this section about log structures of embedding type relative to $t$ :

Theorem 3.12. 1 . There is a canonical class $o \in H^{2}\left(\underline{X}_{e t}, G\right)$ whose vanishing is necessary and sufficient for there to exist a log structure of embedding type relative to $t$.

2. If $o=0$, then the set of isomorphism classes of log structures of embedding type is naturally a torsor under $H^{1}\left(\underline{X}_{e t}, G\right)$.

3. If $\mathcal{M}_{X}$ is a log structure of embedding type, then there is a natural isomorphism of sheaves

$$
\operatorname{Aut}\left(\mathcal{M}_{X}\right) \simeq G .
$$

Proof. It suffices to prove the third statement since we have already seen that any two log structure of embedding type are locally isomorphic. For then the stack over the étale site of $\underline{X}$ whose objects are log structures of embedding type relative to $t$ is a gerbe ([14], IV.2) bound by G. Statements 1 and 2 therefore follows from 3 and ([7], IV.3.4).

Lemma 3.13. Fix a standard chart for $\mathcal{M}_{X}$ as in 3.4, and let $K_{t}=$ $\operatorname{Ker}\left(\times t: \mathcal{O}_{S} \rightarrow \mathcal{O}_{S}\right)$.

1. $\operatorname{Ker}\left(\times X_{i}: \mathcal{O}_{X} \rightarrow \mathcal{O}_{X}\right)=K_{t} \cdot\left(X_{1} \cdots \widehat{X}_{i} \cdots X_{r}\right)$. 
2. The natural map

$$
\bigoplus_{i=1}^{r} K_{t} \cdot\left(X_{1} \cdots \widehat{X}_{i} \cdots X_{r}\right) \rightarrow \sum_{i=1}^{r} K_{t} \cdot\left(X_{1} \cdots \widehat{X}_{i} \cdots X_{r}\right)
$$

is an isomorphism.

Proof. All sheaves involved are quasi-coherent sheaves so it suffices to consider the ring $\mathcal{O}_{S}\left[X_{1}, \ldots, X_{d}\right] /\left(X_{1} \cdots X_{r}-t\right)$. As a module over $\mathcal{O}_{S}$, this ring is isomorphic to

$$
\bigoplus_{\underline{l} \in I} \mathcal{O}_{S} \underline{X}^{\underline{l}}
$$

where $I \subset \mathbb{N}^{d}$ is the subset of $d$-tuples $\left(l_{1}, \ldots, l_{d}\right)$ where $l_{i}=0$ for some $i \in[1, r]$. For each $i \in[1, r]$, let $I_{i} \subset I$ denote the subset of $d$-tuples $\underline{l}$ for which $l_{i}=0$ and $l_{i^{\prime}} \neq 0$ for all $i^{\prime} \in[1, r]-\{i\}$. Then

$$
K_{t} \cdot\left(X_{1} \cdots \widehat{X}_{i} \cdots X_{r}\right) \simeq \bigoplus_{\underline{l} \in I_{i}} K_{t} \underline{X^{\underline{l}}} .
$$

From this 2 follows.

To see 1, observe that multiplication by $X_{i}$ preserves the decomposition in 3.3 if $t=0$, and hence the result is clear in this special case. For the general case, note that the case $t=0$ implies that if $f \in \operatorname{Ker}\left(\times X_{i}\right)$, then there exists $f_{1}$ and $f_{2}$ such that

$$
f=f_{1} X_{1} \cdots \widehat{X}_{i} \cdots X_{r}+f_{2} t=\left(f_{1}+f_{2} X_{i}\right) X_{1} \cdots \widehat{X}_{i} \cdots X_{r}
$$

Since $\underline{X}$ is flat over $\underline{S}$ and $f$ is killed by $X_{i}$, the element $f_{1}+f_{2} X_{i}$ is in $K_{t} \cdot \mathcal{O}_{X}$

Let $\Delta: \mathbb{N} \rightarrow \overline{\mathcal{M}}_{X}$ be the diagonal map and let $\phi: \mathcal{M}_{X} \rightarrow \mathcal{M}_{X}$ be an automorphism. Then for any lifting $\widetilde{\Delta(1)}$ of $\Delta(1)$ to $\mathcal{M}_{X}$

$$
\phi(\widetilde{\Delta(1)})=\lambda(u)+\widetilde{\Delta(1)},
$$

where $u$ is a unit independent of the choice of $\widetilde{\Delta(1)}$. This defines an element $u \in \mathcal{O}_{X}^{*}$. Now if we choose a chart as in 3.4 , then locally the automorphism $\phi$ is induced from solutions $\left\{u_{i}\right\}$ to the equations

$$
U_{i} X_{i}=X_{i}, \quad U_{i} V_{i}=1, \quad i=1, \ldots r .
$$

Hence $u_{i}-1 \in \operatorname{Ker}\left(\times X_{i}: \mathcal{O}_{X} \rightarrow \mathcal{O}_{X}\right)$. From the lemma we conclude that

$$
u_{i}=1+a_{i} X_{1} \cdots \widehat{X}_{i} \cdots X_{r}
$$

where $t a_{i}=0$, and hence

$$
u=\prod_{i=1}^{r}\left(1+a_{i} X_{1} \cdots \widehat{X}_{i} \cdots X_{r}\right)=1+\sum_{i=1}^{r} a_{i} X_{1} \cdots \widehat{X}_{i} \cdots X_{r} \in G .
$$


Conversely, given an element

$$
1+\sum_{i=1}^{r} a_{i} X_{1} \cdots \widehat{X}_{i} \cdots X_{r}
$$

with $t a_{i}=0$ we can define an automorphism of $\mathcal{M}_{X}$ by sending an element $m \in \mathcal{M}_{X}$ with $\alpha(m)=X_{i}$ to $\lambda\left(1+a_{i} X_{1} \cdots \widehat{X}_{i} \cdots X_{r}\right)+m$. This proves the theorem.

Let $H$ be the kernel of $\mathcal{O}_{X}^{*} \rightarrow \mathcal{O}_{D}^{*}$. There is a natural inclusion $G \hookrightarrow H$ which is an isomorphism if $t=0$. The following proposition explains the relationship between theorem 3.12 and the results of F. Kato ([10], theorem 11.7):

Proposition 3.14. 1. The sheaf $\mathcal{E} x t_{\mathcal{O}_{X}}^{1}\left(\Omega_{\underline{X} / \underline{S}}^{1}, \mathcal{O}_{X}\right)$ is an invertible $\mathcal{O}_{D^{-}}$ module.

2. Let $o \in H^{2}\left(\underline{X}_{e t}, G\right)$ be the class constructed in theorem 3.12, and let $o^{\prime} \in H^{1}\left(X_{e t}, \mathcal{O}_{D}^{*}\right)$ be the class of the invertible $\mathcal{O}_{D}$-module

$$
\mathcal{H o m}_{\mathcal{O}_{D}}\left(\mathcal{E} x t_{\mathcal{O}_{X}}^{1}\left(\Omega_{\underline{X} / \underline{S}}^{1}, \mathcal{O}_{X}\right), \mathcal{O}_{D}\right) \text {. }
$$

Then the image of o in $H^{2}\left(\underline{X}_{e t}, H\right)$ is equal to $\delta\left(o^{\prime}\right)$ where

$$
\delta: H^{1}\left(\underline{X}_{e t}, \mathcal{O}_{D}^{*}\right) \longrightarrow H^{2}\left(\underline{X}_{e t}, H\right)
$$

is the map obtained from the exact sequence

$$
0 \longrightarrow H \longrightarrow \mathcal{O}_{X}^{*} \longrightarrow \mathcal{O}_{D}^{*} \longrightarrow 0 .
$$

In particular, if $t=0$ so that $G=H$, then $o=0$ if and only if there exists a line bundle $\mathcal{L}$ on $\underline{X}$ such that

$$
\mathcal{L} \otimes \mathcal{O}_{D} \simeq \mathcal{E} x t^{1}\left(\Omega_{\underline{X} / \underline{S}}^{1}, \mathcal{O}_{X}\right) .
$$

Proof. 1. The question is local so we may assume that we have a standard chart

$$
\underline{X} \longrightarrow \operatorname{Spec}\left(\mathcal{O}_{S}\left[X_{1}, \ldots, X_{d}\right] /\left(X_{1} \cdots X_{r}-t\right)\right)
$$

for $\mathcal{M}_{X}$. Let $R=\mathcal{O}_{S}\left[X_{1}, \ldots, X_{d}\right]$ and let $I$ be the kernel of $R \rightarrow \mathcal{O}_{X}$. Then the conormal sequence gives an exact sequence

$$
I / I^{2} \longrightarrow \Omega_{R / \mathcal{O}_{S}}^{1} \otimes \mathcal{O}_{X} \longrightarrow \Omega_{\underline{X} / \underline{S}}^{1} \longrightarrow 0
$$

Lemma 3.15. The conormal sequence 3.4 is exact on the left.

Proof. The module $\Omega_{R / \mathcal{O}_{S}}^{1} \otimes \mathcal{O}_{X}$ is a free $\mathcal{O}_{X}$-module with basis $\left\{d X_{i}\right\}_{i=1}^{d}$, and $I / I^{2}$ is a free $\mathcal{O}_{X}$-module with basis $X_{1} \cdots X_{r}-t$. The map $I / I^{2} \rightarrow$ $\Omega_{R / \mathcal{O}_{S}}^{1} \otimes \mathcal{O}_{X}$ is the map induced by

$$
X_{1} \cdots X_{r}-t \mapsto \sum_{j=1}^{r} X_{1} \cdots \widehat{X}_{j} \cdots X_{r} d X_{j}
$$


and therefore the kernel can be identified with

$$
\left\{f \in \mathcal{O}_{X} \mid f X_{1} \cdots \widehat{X}_{j} \cdots X_{r}=0, \quad \text { for all } 1 \leq j \leq r\right\} .
$$

Now the kernel of multiplication by $X_{1} \cdots \widehat{X}_{j} \cdots X_{r}$ is equal to $K_{t}^{X} \cdot\left(X_{i}\right)$ where $K_{t}^{X}$ denotes the kernel of multiplication by $t$ on $\mathcal{O}_{X}$, and therefore the kernel of $I / I^{2} \rightarrow \Omega_{R / \mathcal{O}_{S}}^{1} \otimes \mathcal{O}_{X}$ is isomorphic to

$$
\bigcap_{j=1}^{r} K_{t}^{X} \cdot\left(X_{i}\right) .
$$

Using the notation of the proof of lemma 3.13 , the ideal $K_{t}^{X} \cdot\left(X_{i}\right)$ is equal to the ideal

$$
\oplus_{\underline{l} \in L_{i}} K_{t} \underline{X}^{\underline{l}},
$$

where $L_{i}$ denotes the subset of $I$ consisting of elements $\underline{l}$ with $l_{i} \neq 0$. The lemma therefore follows from the statement that

$$
\cap_{i=1}^{r} L_{i}=\emptyset \text {. }
$$

It follows that there is a natural map

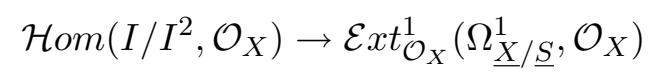

which sits in an exact sequence

$$
\begin{aligned}
& 0 \rightarrow \operatorname{Der}(\underline{X} / \underline{S}) \quad \rightarrow \quad \operatorname{Der}\left(R / \mathcal{O}_{S}\right) \otimes \mathcal{O}_{X} \quad \rightarrow \quad \mathcal{H o m}\left(I / I^{2}, \mathcal{O}_{X}\right) \rightarrow \\
& \rightarrow \mathcal{E} t_{\mathcal{O}_{X}}^{1}\left(\Omega_{\underline{X} / \underline{S}}^{1}, \mathcal{O}_{X}\right) \rightarrow \quad 0 .
\end{aligned}
$$

As mentioned above, the sheaf $I / I^{2}$ is an invertible sheaf with basis the image of $X_{1} \cdots X_{r}-t$, and the map

$$
\operatorname{Der}\left(R / \mathcal{O}_{S}\right) \longrightarrow \mathcal{H o m}\left(I / I^{2}, \mathcal{O}_{X}\right)
$$

is the map which sends $\frac{\partial}{\partial X_{i}}$ to the map

$$
I / I^{2} \rightarrow \mathcal{O}_{X}, \quad X_{1} \cdots X_{r}-t \mapsto X_{1} \cdots \widehat{X_{i}} \cdots X_{r} .
$$

From this it follows that the map in equation 3.5 becomes an isomorphism when tensored with $\mathcal{O}_{D}$.

2. If we view $H^{2}\left(\underline{X}_{e t}, H\right)$ as the group of equivalence classes of gerbes bound by $H$ on $\underline{X}_{e t}$ ([7], IV.3.1.1), then $\delta\left(o^{\prime}\right)$ can be described as the class of the gerbe on $\underline{X}_{e t}$ whose objects are pairs $(\mathcal{L}, \epsilon)$, where $\mathcal{L}$ is an invertible $\mathcal{O}_{X^{-}}$ module and

$$
\epsilon: \mathcal{H o m}_{\mathcal{O}_{X}}\left(\mathcal{L}, \mathcal{O}_{X}\right) \otimes \mathcal{O}_{D} \simeq \mathcal{E} x t_{\mathcal{O}_{X}}^{1}\left(\Omega_{\underline{X} / \underline{S}}^{1}, \mathcal{O}_{X}\right)
$$


is an isomorphism of $\mathcal{O}_{D}$-modules ([7], IV.3.4). Thus to prove the proposition it suffices to exhibit a functor

(stack of log structures of embedding type relative to $t$ )

$$
\begin{gathered}
\downarrow F \\
\text { (stack of pairs }(\mathcal{L}, \epsilon))
\end{gathered}
$$

such that for any two $\log$ structures $\mathcal{M}_{1}$ and $\mathcal{M}_{2}$ of embedding type relative to $t$, the natural map

$$
\operatorname{Isom}\left(\mathcal{M}_{1}, \mathcal{M}_{2}\right) \longrightarrow \operatorname{Isom}\left(F\left(\mathcal{M}_{1}\right), F\left(\mathcal{M}_{2}\right)\right)
$$

induces an isomorphism

$$
\operatorname{Isom}\left(\mathcal{M}_{1}, \mathcal{M}_{2}\right) \times{ }^{G} H \longrightarrow \operatorname{Isom}\left(F\left(\mathcal{M}_{1}\right), F\left(\mathcal{M}_{2}\right)\right) .
$$

To define $F$, suppose $\mathcal{M}_{X}$ is a log structure of embedding type relative to t. We define $\mathcal{L}$ to be the invertible sheaf associated to the torsor of liftings of $\Delta(1) \in \overline{\mathcal{M}}_{X}$ to $\mathcal{M}_{X}$.

To define $\epsilon$ consider first the case when a standard chart is chosen and define $R$ and $I$ as in the proof of part 1 of the proposition. The choice of chart defines trivializations of $I / I^{2}$ and $\mathcal{L}$ and hence an isomorphism $I / I^{2} \simeq \mathcal{L}$. From the map 3.5, we therefore get an isomorphism

$$
\mathcal{H o m}\left(\mathcal{L}, \mathcal{O}_{X}\right) \otimes \mathcal{O}_{D} \simeq \mathcal{E} x t_{\mathcal{O}_{X}}^{1}\left(\Omega_{\underline{X} / \underline{S}}^{1}, \mathcal{O}_{X}\right) .
$$

By a local calculation (which we leave to the reader), this isomorphism is independent of the choices, and hence we get a global isomorphism $\epsilon$.

Define a second sheaf

$$
G_{2}:=\left\{u \in \mathcal{O}_{X}^{*} \mid u t=t\right\} .
$$

Any $\log$ structure of embedding type $\mathcal{M}_{X}$ defines a $G_{2}$-torsor

$$
P_{\mathcal{M}_{X}}:=\left\{m \in \mathcal{M}_{X} \mid m \text { maps to } \Delta(1) \text { in } \overline{\mathcal{M}}_{X} \text { and } \alpha(m)=t\right\} .
$$

By definition, a map $f^{*} \mathcal{M}_{S} \rightarrow \mathcal{M}_{X}$ making $\mathcal{M}_{X}$ a $t$-semi-stable log structure is equivalent to a trivialization of $P_{\mathcal{M}_{X}}$.

From the proof of 3.12.3 it follows that the action of $G$ on $P_{\mathcal{M}_{X}}$ induced by the isomorphism $G \simeq \operatorname{Aut}\left(\mathcal{M}_{X}\right)$ is simply the action obtained from the inclusion $G \hookrightarrow G_{2}$. It follows that a $t$-semi-stable $\log$ structure admits no automorphisms, and hence the presheaf on $\underline{X}_{e t}$

$$
S S^{t}: \underline{U} \mapsto\{\text { isomorphism classes of } t \text {-semi-stable log structures on } \underline{U}\}
$$

is a sheaf. The sheaf $G_{2}$ is naturally identified with the sheaf of automorphisms of $f^{*} \mathcal{M}_{S}$, and hence $G_{2}$ acts naturally on $S S^{t}$ by

$$
\left(f^{*} \mathcal{M}_{S} \longrightarrow \mathcal{M}_{X}\right)^{\sigma}:=\left(f^{*} \mathcal{M}_{S} \stackrel{\sigma}{\longrightarrow} f^{*} \mathcal{M}_{S} \longrightarrow \mathcal{M}_{X}\right) .
$$


Again because any two log structures of embedding type are locally isomorphic, this action of $G_{2}$ is transitive. Moreover, from above we see that this makes $S S^{t}$ a torsor under the sheaf $G_{2} / G$. Thus we obtain:

Theorem 3.16. There is a canonical obstruction $o \in H^{1}\left(\underline{X}, G_{2} / G\right)$ whose vanishing is necessary and sufficient for there to exist a t-semi-stable log structure on $\underline{X}$. If $o=0$, then the set of isomorphism classes of $t$-semistable log structures is canonically a torsor under $H^{0}\left(\underline{X}, G_{2} / G\right)$.

It will be useful to have a better description of the sheaf $G_{2} / G$ :

Proposition 3.17. Let $K_{t} \subset \mathcal{O}_{S}$ (resp. $K_{t}^{X} \subset \mathcal{O}_{X}$ ) be the kernel of multiplication by $t$ on $\mathcal{O}_{S}\left(\right.$ resp. $\left.\mathcal{O}_{X}\right)$, and let $(t) \subset \mathcal{O}_{X}$ be the ideal generated by $t$. Then there is a natural exact sequence

$$
\stackrel{0}{\rightarrow}\left(K_{t}^{X} \cap(t)\right) \otimes \mathcal{O}_{D} \rightarrow G_{2} / G \rightarrow \mathcal{O}_{D}^{*} \rightarrow\left(\mathcal{O}_{D} / K_{t}^{X} \cdot \mathcal{O}_{D}\right)^{*} \rightarrow 0 .
$$

Moreover, the image of o under the natural map

$$
H^{1}\left(\underline{X}_{e t}, G_{2} / G\right) \longrightarrow H^{1}\left(\underline{X}_{e t}, \mathcal{O}_{D}^{*}\right)
$$

is equal to the class of

$$
\mathcal{H o m}_{\mathcal{O}_{D}}\left(\mathcal{E x t}_{\mathcal{O}_{X}}^{1}\left(\Omega_{\underline{X} / \underline{S}}^{1}, \mathcal{O}_{X}\right), \mathcal{O}_{D}\right) .
$$

Proof. To see the exactness of 3.6 we may work locally and hence can assume that a standard chart as in 3.4 has been chosen. Moreover, the exactness of

$$
G_{2} / G \longrightarrow \mathcal{O}_{D}^{*} \longrightarrow\left(\mathcal{O}_{D} / K_{t}^{X} \cdot \mathcal{O}_{D}\right)^{*} \longrightarrow 0
$$

is by definition of $G_{2}$.

Lemma 3.18. 1. $K_{t}^{X} \cap J=\operatorname{Ker}(\times t:(t) \rightarrow(t))+\sum_{i=1}^{r} K_{t}^{X} \cdot\left(X_{1} \cdots \widehat{X}_{i} \cdots X_{r}\right)$.

2. $(t) \cap \sum_{i=1}^{r} K_{t}^{X} \cdot\left(X_{1} \cdots \widehat{X}_{i} \cdots X_{r}\right)=\sum_{i=1}^{r}\left(K_{t}^{X} \cap(t)\right) \cdot\left(X_{1} \cdots \widehat{X}_{i} \cdots X_{r}\right)$.

Proof. Note first of all that since $\underline{X} / \underline{S}$ is flat, $K_{t}^{X}=\mathcal{O}_{X} \otimes_{\mathcal{O}_{S}} K_{t}$.

Since all sheaves involved are quasi-coherent, it suffices to consider the $\operatorname{ring} \mathcal{O}_{S}\left[X_{1}, \ldots, X_{d}\right] /\left(X_{1} \cdots X_{r}-t\right)$. In terms of the decomposition given in equation $3.3, K_{t}^{X}$ is equal to the ideal

$$
\bigoplus_{\underline{l} \in I} K_{t} \underline{X}^{\underline{l}}
$$

and $J$ has a decomposition

$$
\bigoplus_{\underline{l} \in I} \mathcal{O}_{S} t^{\delta(\underline{l})} \underline{X}^{\underline{l}}
$$

where

From this 1 follows.

$$
\delta(\underline{l})=\left\{\begin{array}{cl}
0, & \text { if } l \in I_{i} \text { for some } i \in[1, r] \\
1 & \text { otherwise }
\end{array}\right\} .
$$


To see 2 , note that multiplication by $X_{i}$ on $K_{t}^{X}\left(X_{1} \cdots \widehat{X}_{i} \cdots X_{r}\right)$ is the zero map. Hence

$$
\sum_{i=1}^{r} K_{t}^{X}\left(X_{1} \cdots \widehat{X}_{i} \cdots X_{r}\right)=\bigoplus_{\underline{l} \in I^{\prime}} K_{t} X^{\underline{l}}
$$

where $I^{\prime} \subset I$ is the subset of elements $\underline{l}$ for which there exists exactly one $i \in[1, r]$ such that $l_{i}=0$.

It follows from the first part of the lemma that the kernel of $G_{2} \rightarrow \mathcal{O}_{D}^{*}$ can be described as units $u$ which can be written as

$$
1+f t+\sum_{i=1}^{r} a_{i} X_{1} \cdots \widehat{X}_{i} \cdots X_{r}
$$

where $t^{2} f=t a_{i}=0$. Define a map $K_{t}^{X} \cap(t) \rightarrow G_{2}$ by

$$
f t \mapsto 1+f t .
$$

Then by the second part of the lemma the map induces an injection $\left(K_{t}^{X} \cap\right.$ $\left.(t) \otimes \mathcal{O}_{D}\right) \rightarrow G_{2} / G$, and since any element as in 3.7 can be written as

$$
(1+f t)\left(1+\sum_{i=1}^{r} a_{i} X_{1} \cdots \widehat{X}_{i} \cdots X_{r}\right)
$$

we get the exactness of the rest of the sequence 3.6.

To get the second statement, recall (proof of proposition 3.14) that to any $\log$ structure $\mathcal{M}_{X}$ of embedding type relative to $t$ we associated a pair $(\mathcal{L}, \epsilon)$ where $\mathcal{L}$ is an invertible sheaf and $\epsilon$ is an isomorphism

$$
\epsilon: \mathcal{H o m}_{\mathcal{O}_{X}}\left(\mathcal{L}, \mathcal{O}_{X}\right) \otimes \mathcal{O}_{D} \simeq \mathcal{E} x t_{\mathcal{O}_{X}}^{1}\left(\Omega_{\underline{X} / \underline{S}}^{1}, \mathcal{O}_{X}\right)
$$

Now from the proof of proposition 3.14, a map $f^{*} \mathcal{M}_{S} \rightarrow \mathcal{M}_{X}$ making $\mathcal{M}_{X}$ $t$-semi-stable defines a trivialization of $\mathcal{L}$. Hence we get a map

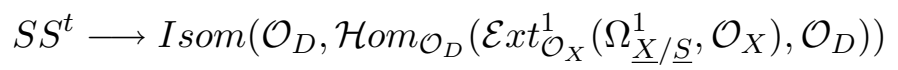

compatible with the actions of $G_{2} / G$ and $\mathcal{O}_{D}^{*}$.

\section{A theorem about efFeCtivity of Formal LOG StRUCtURES}

Theorem 4.1. Suppose $A$ is the completion at a point of a scheme of finite type over an excellent Dedeking ring, and let $f: \underline{X} \rightarrow \operatorname{Spec}(A)$ be a proper morphism of schemes such that all the fibers of $\underline{X}$ are semi-stable varieties in the sense of definition 1.1. For each $n \geq \overline{0}$, let $A_{n}=A / \mathfrak{m}_{A}^{n+1}$ and $\underline{X}_{n}:=\underline{X} \times_{\operatorname{Spec}(A)} \operatorname{Spec}\left(A_{n}\right)$ be the reductions and let $f_{n}: \underline{X}_{n} \rightarrow \operatorname{Spec}\left(A_{n}\right)$ be the natural map. Fix a log structure $\mathcal{M}_{A}$ on $\operatorname{Spec}(A)$ and let $\mathcal{M}_{A_{n}}$ be the pullback of $\mathcal{M}_{A}$ to $\operatorname{Spec}\left(A_{n}\right)$. Then any compatible family of pairs $\left(\mathcal{M}_{n}, f_{n}^{b}\right)$, 
where $\mathcal{M}_{n}$ is a log structure on $\underline{X}_{n}$ and $f_{n}^{b}: f_{n}^{*} \mathcal{M}_{A_{n}} \rightarrow \mathcal{M}_{n}$ is a morphism of log structures making the map

$$
\left(f_{n}, f_{n}^{b}\right):\left(\underline{X}_{n}, \mathcal{M}_{n}\right) \longrightarrow\left(\operatorname{Spec}\left(A_{n}\right), \mathcal{M}_{A_{n}}\right)
$$

log smooth, vertical, and integral, is induced by a unique pair $\left(\mathcal{M}, f^{b}\right)$, where $\mathcal{M}$ is a log structure on $\underline{X}$ and $f^{*} \mathcal{M}_{A} \rightarrow \mathcal{M}$ is a morphism of log structures making

$$
\left(f, f^{b}\right):(\underline{X}, \mathcal{M}) \longrightarrow\left(\operatorname{Spec}(A), \mathcal{M}_{A}\right)
$$

log smooth, vertical, and integral.

Proof. By theorem 2.6, it suffices to consider the case when all the morphisms $\left(f_{n}, f_{n}^{b}\right)$ are special in the sense of definition 2.5. Moreover, by descent theory we may assume that the residue field $A / \mathfrak{m}_{A}$ is separably closed.

Let $\Gamma$ denote the set of connected components of the singular locus of the closed fiber $\underline{X}_{0}$. Then by the definition of a special morphism (2.5) there is a canonical isomorphism $\mathbb{N}^{\Gamma} \simeq \overline{\mathcal{M}}_{A_{0}}$. Since $A$ is strictly henselian and local, there is an isomorphism

$$
H^{0}\left(\operatorname{Spec}(A), \overline{\mathcal{M}}_{A}\right) \simeq \overline{\mathcal{M}}_{A_{0}} .
$$

Moreover, $H^{1}\left(\operatorname{Spec}(A), \mathcal{O}_{A}^{*}\right)=0$, and hence from the exact sequence

$$
0 \longrightarrow \mathcal{O}_{A}^{*} \longrightarrow \mathcal{M}_{A}^{g p} \longrightarrow \overline{\mathcal{M}}_{A}^{g p} \longrightarrow 0
$$

we see that the map

$$
H^{0}\left(\operatorname{Spec}(A), \mathcal{M}_{A}\right) \longrightarrow H^{0}\left(\operatorname{Spec}(A), \overline{\mathcal{M}}_{A}\right)
$$

is surjective. Therefore,there exists a chart $\beta: \mathbb{N}^{\Gamma} \rightarrow \mathcal{M}_{A}$ inducing $\beta_{0}$. By the construction in the proof of theorem 2.6, there is a canonical decomposition for all $n$

$$
\mathcal{M}_{n} \simeq \oplus_{\gamma \in \Gamma, \mathcal{O}_{X_{n}}^{*}} \mathcal{M}_{n}^{\gamma}
$$

Let $t_{\gamma}$ be the image of the standard generator of $\mathbb{N}^{\gamma} \hookrightarrow \mathbb{N}^{\Gamma}$ under the map $\mathbb{N}^{\Gamma} \rightarrow \mathcal{M}_{A} \rightarrow A$. Then each $\mathcal{M}_{n}^{\gamma}$ is a $t_{\gamma}$-semi-stable log structure on any open set of $\underline{X}_{n}$ not intersecting the components $\Gamma-\{\gamma\}$. We denote by $\mathcal{M}_{A}^{\gamma}$ the $\log$ structure defined by the map $\mathbb{N} \rightarrow A, 1 \mapsto t_{\gamma}$.

Next we claim that for any closed point $x_{0} \in X$ in the closed fiber, there exists an étale neighborhood $\underline{U}$ of $x_{0}$ and an étale morphism

$$
\underline{U} \longrightarrow \operatorname{Spec}\left(A\left[X_{1}, \ldots, X_{d}\right] /\left(X_{1} \cdots X_{r}-t_{\gamma}\right)\right)
$$

for some $d, r$ and $\gamma$. To see this, choose an affine étale neighborhood $U_{0}$ of $x_{0}$ and a standard chart (see 3.4)

$$
\underline{U}_{0} \longrightarrow \operatorname{Spec}\left(A_{0}\left[X_{1}, \ldots, X_{d}\right] /\left(X_{1} \cdots X_{r}\right)\right)
$$

for $\mathcal{M}_{X_{0}}^{\gamma}$. For each integer $n$, there is a unique étale $\underline{X}_{n}$-scheme $\underline{U}_{n}$ reducing to $\underline{U}_{0}$. We inductively lift the standard chart over $\underline{U}_{0}$ to a compatible family of standard charts over $\underline{U}_{n}$. 
For each $n$, view $\operatorname{Spec}\left(A_{n}\left[X_{1}, \ldots, X_{d}\right] /\left(X_{1} \cdots X_{r}-t_{\gamma}\right)\right)$ as a smooth log scheme over $\left(\operatorname{Spec}\left(A_{n}\right), \mathcal{M}_{A_{n}}^{\gamma}\right)$, and note that giving a lifting to $\underline{U}_{n}$ of a standard chart over $\underline{U}_{n-1}$ is equivalent to finding a dotted arrow filling in the diagram of log schemes

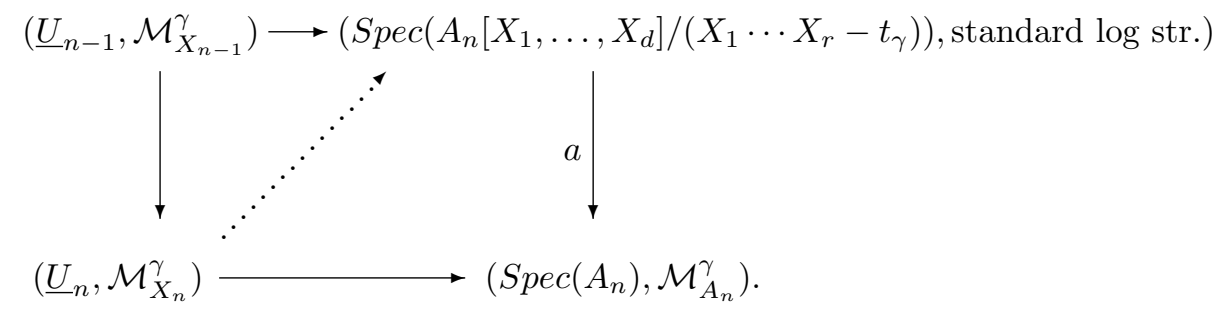

This is because a map between flat schemes over $A_{n}$ is étale if and only if its reduction to $A_{0}$ is étale. Since the map labelled $a$ in the diagram is log smooth, such a dotted arrow exists. It follows from the Artin approximation theorem $([2], 2.5)$ that there exists an étale neighborhood $\underline{U}$ of $x_{0}$ and a map as in 4.1 approximating our formal solution in $\widehat{\mathcal{O}}_{X, x_{0}}$ obtained from the charts over the $\underline{U}_{n}$. Since the resulting map is formally étale (and hence étale) at $x_{0}$, we obtain the claim.

To prove the theorem, it suffices to show that there exists a $t_{\gamma}$-semistable $\log$ structure on $\underline{X}$ inducing $\mathcal{M}_{n}^{\gamma}$. The fact that $\underline{X} / A$ is locally $t$ semi-stable for some $t$ implies that the singular locus of $\underline{X}$ has a natural scheme structure: define $\underline{X}^{\text {sing }}$ to be the closed subscheme defined by the annihilator of the sheaf

$$
\mathcal{E} x t^{1}\left(\Omega_{\underline{X} / A}^{1}, \mathcal{O}_{X}\right) .
$$

If we choose a map as in 4.1 , then $\underline{X}^{\text {sing }}$ is by proposition 3.14 the closed subscheme defined by the ideal

$$
\left(\ldots, X_{1} \cdots \widehat{X}_{i} \cdots X_{r}, \ldots\right)_{i=1}^{r} \text {. }
$$

From this local description it also follows that the reduction of $\underline{X}^{\text {sing }}$ to $\underline{X}_{n}$ is equal to the disjoint union of the $D_{\gamma}^{n}$ where $D_{\gamma}^{n}$ is the closed subscheme defined in 3.2 applied to any open set of $\underline{X}_{n}$ not containing the other components $\Gamma-\{\gamma\}$. Now by the Grothendieck existence theorem ([6], III.5), the functor which sends a closed subscheme of $\underline{X}$ to its reductions induces a bijection

$$
\text { (closed subschemes of } \underline{X} \text { ) }
$$

(compatible families of closed subschemes of the $\underline{X}_{n}$ ).

Therefore, $\underline{X}^{\text {sing }}$ has a canonical decomposition

$$
\underline{X}^{\text {sing }}=\coprod_{\gamma \in \Gamma} D_{\gamma}
$$


where $D_{\gamma}$ reduces to $D_{\gamma}^{n}$ over $\underline{X}_{n}$. Now to construct a $\log$ structure $\mathcal{M}^{\gamma}$ inducing the $\mathcal{M}_{n}^{\gamma}$, we may as well replace $\underline{X}$ by $\underline{X}-\cup_{\gamma^{\prime} \neq \gamma} D_{\gamma^{\prime}}$. We apply the results of section 3 to $\underline{U}$.

Let $G, G_{2}$, and $D$ (resp. $G^{n}, G_{2}^{n}$, and $D^{n}$ ) be as in section 3 applied to $\underline{U} / A$ (resp. $\underline{U}_{n} / A_{n}$ ). Note that $D$ is proper over $\operatorname{Spec}(A)$, flat over $\operatorname{Spec}\left(A / t_{\gamma}\right)$, and that there is a natural isomorphism

$$
D^{n} \simeq D \times_{\operatorname{Spec}(A)} \operatorname{Spec}\left(A_{n}\right) .
$$

By theorem 3.16, it suffices to show that the map

$$
H^{1}\left(\underline{X}, G_{2} / G\right) \longrightarrow \lim _{\longleftarrow} H^{1}\left(\underline{X}_{n}, G_{2}^{n} / G^{n}\right)
$$

is injective, and that the map

$$
H^{0}\left(\underline{X}, G_{2} / G\right) \longrightarrow \lim _{\longleftarrow} H^{0}\left(\underline{X}_{n}, G_{2}^{n} / G^{n}\right)
$$

is bijective.

Lemma 4.2. For any $A /\left(t_{\gamma}\right)$-module $M$ of finite type, the natural map

$$
M \longrightarrow H^{0}\left(\underline{U}, M \otimes \mathcal{O}_{D}\right)
$$

is an isomorphism.

Proof. Since $D$ is proper over $\underline{A}$, the natural map

$H^{0}\left(\underline{U}, M \otimes \mathcal{O}_{D}\right) \longrightarrow \lim _{n} H^{0}\left(\underline{U}, M \otimes \mathcal{O}_{D^{n}}\right) \simeq{\underset{\lim }{n}}^{0} H^{0}\left(\underline{U}, M \otimes A_{n} \otimes_{A_{n}} \mathcal{O}_{D^{n}}\right)$

is an isomorphism by ([6], III.5.1.2). Therefore, it suffices to consider the case when $A$ is an Artin local ring. Since $A$ is noetherian and artinian, there exists a filtration of $M$ by $A / t_{\gamma}$-modules

$$
0=M_{r} \subset \cdots \subset M_{2} \subset M_{1}=M
$$

such that for each $i, M_{i} / M_{i+1} \simeq A / \mathfrak{m}_{A}$. Now if the results holds for $M=$ $A / \mathfrak{m}_{A}$ and $M_{i}$, then the snake lemma applied to the commutative diagram

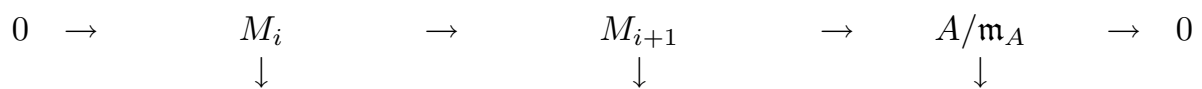

$$
\begin{aligned}
& 0 \rightarrow H^{0}\left(\underline{U}, M_{i} \otimes \mathcal{O}_{D}\right) \rightarrow H^{0}\left(\underline{U}, M_{i+1} \otimes \mathcal{O}_{D}\right) \rightarrow H^{0}\left(\underline{U}, \mathcal{O}_{D^{0}}\right)
\end{aligned}
$$

obtained from the exact sequence ( $D$ is flat over $A / t_{\gamma}$ )

$$
0 \longrightarrow M_{i} \otimes \mathcal{O}_{D} \longrightarrow M_{i+1} \otimes \mathcal{O}_{D} \longrightarrow \mathcal{O}_{D^{0}} \longrightarrow 0
$$

yields the results for $M_{i+1}$. Therefore, it suffices to consider the case when $M=A / \mathfrak{m}_{A}$. In this case we may even base change to an algebraic closure of $A / \mathfrak{m}_{A}$, and hence we may assume that $A / \mathfrak{m}_{A}$ is algebraically closed. But then $D^{0}$ is a reduced proper scheme over an algebraically closed field, and hence $H^{0}\left(D^{0}, \mathcal{O}_{D^{0}}\right)=A / \mathfrak{m}_{A}$. 
Let $K_{t_{\gamma}}$ (resp. $K_{t_{\gamma}}^{n}$ ) be the kernel of multiplication by $t_{\gamma}$ on $A$ (resp. on $A_{n}$ ), and let $\mathcal{F}$ (resp. $\mathcal{F}^{n}$ ) be the image of the map $G_{2} \rightarrow \mathcal{O}_{D}^{*}$ (resp. $\left.G_{2}^{n} \rightarrow \mathcal{O}_{D^{n}}^{*}\right)$. Note that since $\underline{U}$ is flat over $A$, we have in the notation of proposition 3.17

$$
\left(K_{t_{\gamma}} \cap t_{\gamma} A\right) \otimes_{A} \mathcal{O}_{D} \simeq\left(K_{t_{\gamma}}^{U} \cap t_{\gamma} \mathcal{O}_{\underline{U}}\right) \otimes_{\mathcal{O}_{\underline{U}}} \mathcal{O}_{D}
$$

and

$$
\left(K_{t_{\gamma}}^{n} \cap t_{\gamma} A_{n}\right) \otimes_{A_{n}} \mathcal{O}_{D^{n}} \simeq\left(K_{t_{\gamma}}^{U_{n}} \cap t_{\gamma} \mathcal{O}_{\underline{U}_{n}}\right) \otimes_{\mathcal{O}_{\underline{U}}} \mathcal{O}_{D^{n}}
$$

\section{Corollary 4.3.}

$$
H^{0}(\underline{U}, \mathcal{F})=\left\{u \in A^{*} \mid u t_{\gamma}=t_{\gamma}\right\} /\left\{u \in A^{*} \mid 1-u \in K_{t_{\gamma}} \cap t_{\gamma} A\right\}
$$

and

$$
H^{0}\left(\underline{U}_{n}, \mathcal{F}^{n}\right)=\left\{u \in A_{n}^{*} \mid u t_{\gamma}=t_{\gamma}\right\} /\left\{u \in A_{n}^{*} \mid 1-u \in K_{t_{\gamma}}^{n} \cap t_{\gamma} A_{n}\right\} .
$$

Proof. Since $\underline{X}$ is flat over $A$, there are natural isomorphisms

$$
\mathcal{O}_{D} / K_{t}^{X} \mathcal{O}_{D} \simeq \mathcal{O}_{D} \otimes_{A / t_{\gamma}}\left(\left(A / t_{\gamma}\right) / K_{t_{\gamma}} \cdot\left(A / t_{\gamma}\right)\right)
$$

and

$$
\mathcal{O}_{D^{n}} / K_{t}^{X_{n}} \mathcal{O}_{D^{n}} \simeq \mathcal{O}_{D^{n}} \otimes_{A_{n} / t_{\gamma}}\left(\left(A_{n} / t_{\gamma}\right) / K_{t_{\gamma}}^{n} \cdot\left(A_{n} / t_{\gamma}\right)\right)
$$

Hence lemma 4.2 and the exact sequences

$$
\begin{aligned}
0 \longrightarrow \mathcal{F} \longrightarrow \mathcal{O}_{D}^{*} \longrightarrow\left(\mathcal{O}_{D} / K_{t}^{X} \mathcal{O}_{D}\right)^{*} \longrightarrow 0 \\
0 \longrightarrow \mathcal{F}^{n} \longrightarrow \mathcal{O}_{D^{n}}^{*} \longrightarrow\left(\mathcal{O}_{D^{n}} / K_{t}^{X_{n}} \mathcal{O}_{D^{n}}\right)^{*} \longrightarrow 0
\end{aligned}
$$

give exact sequences

$$
\begin{gathered}
0 \longrightarrow H^{0}(\underline{U}, \mathcal{F}) \longrightarrow\left(A / t_{\gamma}\right)^{*} \longrightarrow\left(\left(A / t_{\gamma}\right) / K_{t_{\gamma}} \cdot\left(A / t_{\gamma}\right)\right)^{*} \\
\left.0 \longrightarrow H^{0}\left(\underline{U}_{n}, \mathcal{F}^{n}\right) \longrightarrow\left(A_{n} / t_{\gamma}\right)^{*} \longrightarrow\left(A_{n} / t_{\gamma}\right) / K_{t_{\gamma}}^{n} \cdot\left(A_{n} / t_{\gamma}\right)\right)^{*} .
\end{gathered}
$$

From this the corollary follows.

The corollary implies that $H^{0}\left(\underline{U}, G_{2} / G\right) \rightarrow H^{0}(\underline{U}, \mathcal{F})$ is surjective, and hence there is a commutative diagram (4.6)

$$
\begin{aligned}
& 0 \rightarrow K_{t_{\gamma}} \cap t_{\gamma} A \quad \rightarrow \quad H^{0}\left(\underline{U}, G_{2} / G\right) \quad \rightarrow \quad H^{0}(\underline{U}, \mathcal{F}) \quad \rightarrow \quad 0 \\
& 0 \rightarrow \underset{\lim }{\longleftarrow} K_{t_{\gamma}}^{n} \cap t_{\gamma} A_{n} \rightarrow \underset{\lim }{\longleftarrow} H^{0}\left(\underline{U}_{n}^{\downarrow}, G_{2}^{n} / G^{n}\right) \rightarrow \underset{l}{\downarrow} H^{0}\left(\underline{U}_{n}, \mathcal{F}^{n}\right)
\end{aligned}
$$

with exact rows. The following lemma implies that the map 4.3 is bijective.

Lemma 4.4. Let $L_{t_{\gamma}}^{n}:=\operatorname{Im}\left(K_{t_{\gamma}} \cap t_{\gamma} A \rightarrow K_{t_{\gamma}}^{n} \cap t_{\gamma} A_{n}\right)$. Then there exists integers $n_{0}$ and $n_{0}^{\prime}$ such that:

1. For each $n$, the map

$$
\left(K_{t_{\gamma}} \cap t_{\gamma} A\right) \otimes A_{n+n_{0}} \longrightarrow\left(K_{t_{\gamma}} \cap t_{\gamma} A\right) \otimes A_{n}
$$

factors through $L_{t_{\gamma}}^{n+n_{0}}$. 
2. For every $n$, the map

$$
K_{t_{\gamma}}^{n+n_{0}^{\prime}} \cap t_{\gamma} A_{n+n_{0}^{\prime}} \longrightarrow K_{t_{\gamma}}^{n}
$$

factors through $L_{t_{\gamma}}^{n+n_{0}^{\prime}}$.

3. The natural map

$$
H^{0}(\underline{U}, \mathcal{F}) \longrightarrow \lim _{\longleftarrow} H^{0}\left(\underline{U}_{n}, \mathcal{F}^{n}\right)
$$

is an isomorphism.

Proof. By the Artin-Rees lemma there exists an integer $n_{0}$ such that

$$
K_{t_{\gamma}} \cap t_{\gamma} A \cap \mathfrak{m}_{A}^{n+n_{0}} \subset \mathfrak{m}_{A}^{n}\left(K_{t_{\gamma}} \cap t_{\gamma} A\right)
$$

for every $n$. From this 1 follows.

Similarly, the Artin-Rees lemma implies that there exists an integer $n_{0}^{\prime}$ such that

$$
\left(t_{\gamma}\right)^{2} \cap \mathfrak{m}_{A}^{n+n_{0}^{\prime}} \subset \mathfrak{m}_{A}^{n}\left(t_{\gamma}\right)^{2} .
$$

Suppose $t_{\gamma} f \in K_{t_{\gamma}}^{n+n_{0}^{\prime}} \cap t_{\gamma} A_{n+n_{0}^{\prime}}$ and let $\tilde{f} \in A$ be a lifting of $f$ to $A$. Then $t_{\gamma}^{2} \tilde{f} \in \mathfrak{m}_{A}^{n+n_{0}+1}$, and hence $t_{\gamma}^{2} \tilde{f} \in \mathfrak{m}_{A}^{n+1}\left(t_{s}\right)^{2}$. Let $g \in \mathfrak{m}_{A}^{n+1}$ be an element such that $t_{\gamma}^{2} \tilde{f}=t_{\gamma}^{2} g$. Then $t_{\gamma}(g-f) \in K_{t_{\gamma}} \cap\left(t_{\gamma}\right)$, and hence the image of

$$
K_{t_{\gamma}}^{n+n_{0}^{\prime}} \cap t_{\gamma} A_{n+n_{0}^{\prime}} \longrightarrow K_{t_{\gamma}}^{n}
$$

is contained in $L_{t_{\gamma}}^{n+n_{0}}$. This implies 2 .

To prove part 3 , let $\Lambda_{n}$ be the group

$$
\left\{u \in A_{n}^{*} \mid 1-u \in K_{t_{\gamma}}^{n} \cap t_{\gamma} A_{n}\right\} .
$$

Then by corollary 4.3 there are exact sequences

$$
0 \rightarrow \Lambda_{n} \rightarrow\left\{u \in A_{n}^{*} \mid u t_{\gamma}=t_{\gamma}\right\} \quad \rightarrow \quad H^{0}\left(\underline{U}_{n}, \mathcal{F}^{n}\right) \quad \rightarrow \quad 0,
$$

and by part 2 the $\Lambda_{n}$ satisfy the Mittag-Leffler condition. Hence if $\Lambda$ denotes the group

$$
\left\{u \in A^{*} \mid 1-u \in K_{t_{\gamma}} \cap t_{\gamma} A\right\},
$$

there is a morphism of exact sequences

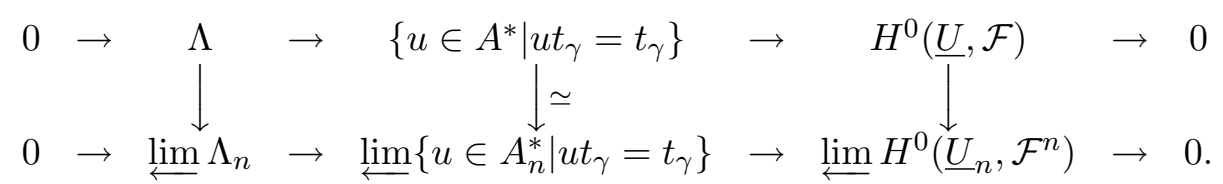

Part 3 of the lemma now follows because part 1 implies that the left vertical arrow is an isomorphism. 
The preceding lemma also implies that the natural map

$$
H^{1}\left(\underline{U},\left(K_{t_{\gamma}} \cap t_{\gamma} A\right) \otimes \mathcal{O}_{D}\right) \rightarrow \underset{\lim }{\longleftarrow} H^{1}\left(\underline{U}_{n},\left(K_{t_{\gamma}}^{n} \cap t_{\gamma} A_{n}\right) \otimes \mathcal{O}_{D^{n}}\right)
$$

is an isomorphism. By corollary 4.3, the maps

$$
\begin{gathered}
H^{1}\left(\underline{U},\left(K_{t_{\gamma}} \cap t_{\gamma} A\right) \otimes \mathcal{O}_{D}\right) \rightarrow H^{1}\left(\underline{G}_{2} / G\right) \\
H^{1}\left(\underline{U}_{n},\left(K_{t_{\gamma}}^{n} \cap t_{\gamma} A_{n}\right) \otimes \mathcal{O}_{D^{n}}\right) \rightarrow H^{1}\left(\underline{G}_{2}^{n} / G^{n}\right)
\end{gathered}
$$

are injective. From this and the diagram

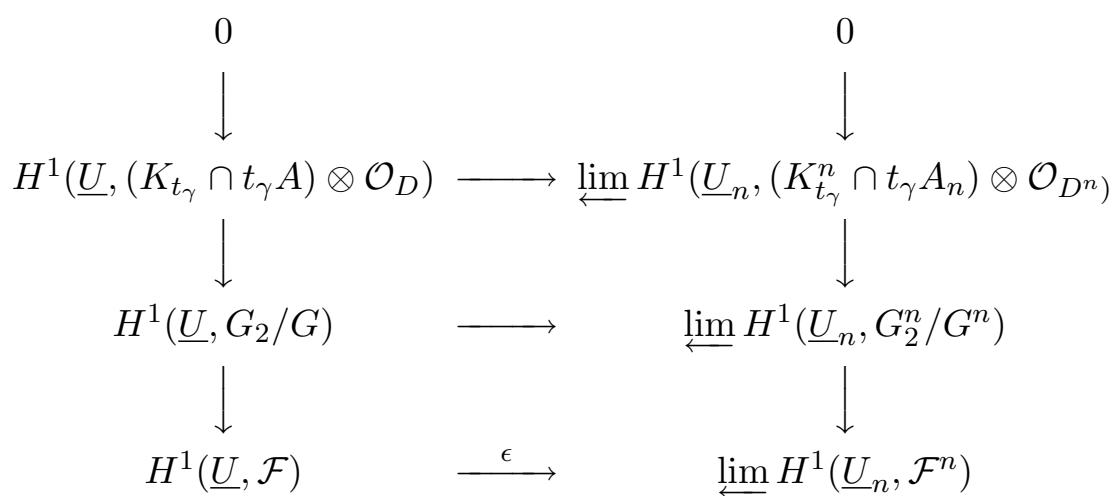

we see that to verify that 4.2 is injective it suffices to show that the map labelled $\epsilon$ in the diagram above is injective.

The exact sequences 4.4 and 4.5 together with lemma 4.2 imply that there is a commutative diagram

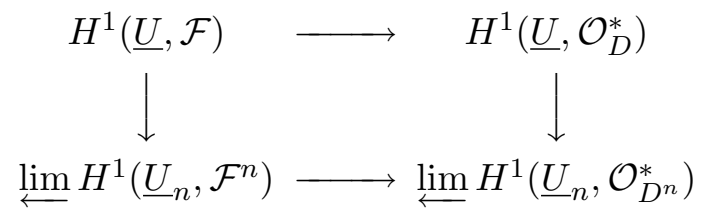

where the horizontal arrows are injections. Therefore, to verify that $\epsilon$ is injective it is enough to verify that the map

$$
H^{1}\left(\underline{U}, \mathcal{O}_{D}^{*}\right) \rightarrow \varliminf_{\longleftarrow} H^{1}\left(\underline{U}_{n}, \mathcal{O}_{D^{n}}^{*}\right)
$$

is injective. This follows from ([6], III.5.1.6), and hence the proof of theorem 4.1 is complete.

\section{Proof of Theorem 1.2}

We now prove theorem 1.2. Let $I V L S$ be the fibered category over the category of $\underline{S}$-schemes which to any $\underline{T} \rightarrow \underline{S}$ associates the category of triples $\left(\mathcal{M}_{T}, \mathcal{M}_{X_{T}}, f_{T}^{b}\right)$ where $\mathcal{M}_{T}$ is a log structure on $\underline{T}, \mathcal{M}_{X_{T}}$ is a log structure on $\underline{X} \times \underline{S} \underline{T}$, and $f_{T}^{b}: p r_{2}^{*} \mathcal{M}_{T} \rightarrow \mathcal{M}_{X_{T}}$ is a morphism of log structures making

$$
\left(f, f^{b}\right):\left(\underline{X}_{T}, \mathcal{M}_{X_{T}}\right) \longrightarrow\left(\underline{T}, \mathcal{M}_{T}\right)
$$


a special morphism in the sense of definition 2.5. There is a natural log structure on the fibered category $\underline{I V L S}$ (see the introduction for the definition of a $\log$ structure on a fibered category)

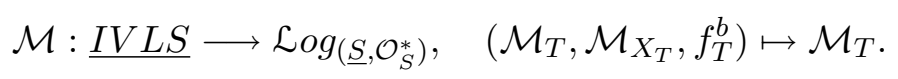

Since $\underline{I V L S}$ classifies sheaves for the étale topology, $\underline{I V L S}$ is a stack over $\underline{S}$, and by $([16], 4.3) \underline{I V L S}$ is limit preserving. By theorem 2.6, if $\left(\underline{T}, \mathcal{M}_{T}^{\prime}\right)$ is a $\log$ scheme, then to give an element of $I V L S$ over $\left(\underline{T}, \mathcal{M}_{T}^{\prime}\right)$ is equivalent to giving an object $\left(\mathcal{M}_{T}, \mathcal{M}_{X_{T}}, f_{T}^{b}\right)$ of $\underline{I V L S}$ over $\underline{T}$ together with a morphism of $\log$ structures $\mathcal{M}_{T} \rightarrow \mathcal{M}_{T}^{\prime}$. Hence to prove theorem 1.2, it suffices to show that $\underline{I V L S}$ is representable by a scheme, and that the map $\underline{I V L S} \rightarrow \underline{S}$ is a monomorphism of finite type.

Now any algebraic space which admits a quasi-finite map to a scheme is also a scheme $([4], 3.3)$, and hence it suffices to show that $\underline{I V L S}$ is an algebraic space. We proceed in two steps (theorems 5.1 and 5.4).

Theorem 5.1. IVLS is relatively representable and IVLS is equivalent to the stack defined by a sub-functor of $\underline{S}$.

Proof. Suppose $\left(\mathcal{M}_{T}, \mathcal{M}_{X_{T}}, f_{T}^{b}\right)$ and $\left(\mathcal{M}_{T}^{\prime}, \mathcal{M}_{X_{T}}^{\prime}, f_{T}^{b \prime}\right)$ are two objects of $\underline{I V L S}$ over some scheme $\underline{T}$. What has to be shown is that there exists unique isomorphisms $\sigma: \overline{\mathcal{M}}_{T} \simeq \mathcal{M}_{T^{\prime}}$ and $\phi: \mathcal{M}_{X_{T}} \simeq \mathcal{M}_{X_{T}}^{\prime}$ such that the diagram

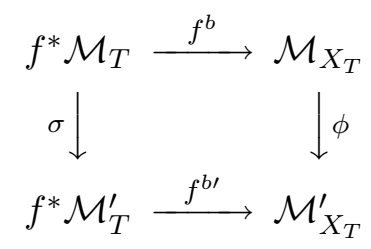

commutes. Let us begin by showing the uniqueness of the pair $(\sigma, \phi)$. For this it suffices to consider the case when $\underline{T}=\operatorname{Spec}(A)$ is a complete local ring.

Let $S S\left(\left(\underline{T}, \mathcal{M}_{T}\right)\right)$ be the set of isomorphism classes of pairs $\left(\mathcal{M}_{X}, f^{b}\right)$, where $\mathcal{M}_{X}$ is a log structure on $\underline{X}$ and $f^{b}: f^{*} \mathcal{M}_{T} \rightarrow \mathcal{M}_{X}$ is a morphism of $\log$ structures making

$$
\left(\underline{X}, \mathcal{M}_{X}\right) \longrightarrow\left(\underline{T}, \mathcal{M}_{T}\right)
$$

a special morphism (in the sense of definition 2.5).

Let

$$
\rho: \operatorname{Aut}\left(\mathcal{M}_{T}\right) \longrightarrow \operatorname{End}\left(S S\left(\left(\underline{T}, \mathcal{M}_{T}\right)\right)\right)
$$

be the action given by

$$
\left(f^{*} \mathcal{M}_{T} \longrightarrow \mathcal{M}_{X}\right)^{\rho(\sigma)}:=\left(f^{*} \mathcal{M}_{T} \stackrel{\sigma}{\longrightarrow} f^{*} \mathcal{M}_{T} \longrightarrow \mathcal{M}_{X}\right) .
$$

The following proposition implies the uniqueness of $(\sigma, \phi)$. 
Proposition 5.2. 1. For a pair $\left(\mathcal{M}_{X}, f^{b}\right)$ as above, there are no automorphisms of $\mathcal{M}_{X}$ compatible with the map $f^{b}$.

2. The action $\rho$ on $S S\left(\left(\underline{T}, \mathcal{M}_{T}\right)\right)$ is simply transitive.

Proof. 1. It suffices by descent theory to consider the case when $A$ has separably closed residue field. In this case, any object $\left(\mathcal{M}_{X}, f^{b}\right) \in S S(\operatorname{Spec}(A))$ has a canonical decomposition

$$
\mathcal{M}_{X} \simeq \oplus_{\gamma \in \Gamma, \mathcal{O}_{X}^{*}} \mathcal{M}_{X}^{\gamma}
$$

where $\Gamma$ denotes the set of connected components of the singular locus of the closed fiber. Moreover, $\left(\mathcal{M}_{X}, f^{b}\right)$ induces a decomposition

$$
\mathcal{M}_{T} \simeq \oplus_{\gamma \in \Gamma, \mathcal{O}_{T}^{*}} \mathcal{M}_{T}^{\gamma} \text {. }
$$

This implies 1 for any automorphism of $\mathcal{M}_{X}$ must preserve the above decompositions and hence induces automorphisms of the $\log$ structures $\mathcal{M}_{X}^{\gamma}$ over $\mathcal{M}_{T}^{\gamma}$. But by the discussion preceding theorem 3.16, there are no such automorphisms other than the identity.

2. We first reduce to the case when $A$ is an artin local ring with separably closed residue field. Let $\mathcal{M}_{T_{n}}$ be the reduction of $\mathcal{M}_{T}$ to $A_{n}:=A / \mathfrak{m}_{A}^{n+1}$. By theorem 4.1 and the fact that $\operatorname{Aut}\left(\mathcal{M}_{T}\right)$ is representable, the natural maps

$$
\operatorname{Aut}\left(\mathcal{M}_{T}\right) \longrightarrow \lim _{\longleftarrow} \operatorname{Aut}\left(\mathcal{M}_{T_{n}}\right), \quad S S(\operatorname{Spec}(A)) \longrightarrow \varliminf_{\longleftarrow}^{\lim } S S\left(\operatorname{Spec}\left(A_{n}\right)\right)
$$

are bijective, and hence it suffices to consider the case when $A$ is an artin local ring. Moreover by 1 , both $\operatorname{Aut}\left(\mathcal{M}_{T}\right)$ and $S S(\operatorname{Spec}(A))$ are sheaves on the étale site, so we can by descent theory assume that $A$ has separably closed residue field.

In this case, any object $\left(\mathcal{M}_{X}, f^{b}\right) \in S S(\operatorname{Spec}(A))$ has a canonical decomposition as in 5.1 and induces a decomposition of $\mathcal{M}_{T}$ as in 5.2.

Suppose given two objects

$$
\left(\mathcal{M}_{X}, f^{b}\right),\left(\mathcal{M}_{X}^{\prime}, f^{b \prime}\right) \in S S(\operatorname{Spec}(A))
$$

inducing two decompositions

$$
\mathcal{M}_{T} \simeq \oplus_{\gamma \in \Gamma, \mathcal{O}_{T}^{*}} \mathcal{M}_{T}^{\gamma} \simeq \oplus_{\gamma \in \Gamma, \mathcal{O}_{T}^{*}} \mathcal{M}_{T}^{\gamma \prime}
$$

The following lemma, applied to the completion of $\mathcal{O}_{X}$ at a point in $\gamma$, shows that $\mathcal{M}_{T}^{\gamma}$ and $\mathcal{M}_{T}^{\gamma \prime}$ are isomorphic as log structures (not as sub-log structures of $\mathcal{M}_{T}$ ):

Lemma 5.3. Suppose $t, t^{\prime} \in A$ are two elements such that there exists $d, d^{\prime}, r, r^{\prime} \in \mathbb{Z}$ and an isomorphism

$$
\phi: A\left[\left[X_{1}, \ldots, X_{d}\right]\right] /\left(X_{1} \cdots X_{r}-t\right) \simeq A\left[\left[Y_{1}, \ldots, Y_{d^{\prime}}\right]\right] /\left(Y_{1} \cdots Y_{r^{\prime}}-t^{\prime}\right) .
$$

Then there exists a unit $u \in A^{*}$ such that $u t=t^{\prime}$. 
Proof. If the result holds with $A$ replaced by $A /\left(t^{\prime}\right)$, then $t \in\left(t^{\prime}\right)$ and by symmetry $(t)=\left(t^{\prime}\right)$. Therefore it suffices to consider the case when $t^{\prime}=0$. Moreover, in this case it suffices to consider the reduction modulo $t^{2}$. Write

$$
\phi\left(X_{i}\right)=c_{i}+f(\underline{Y}), \quad i=1, \ldots, r .
$$

where $c_{i} \in A$ and $f(\underline{Y})$ consists of terms of degree $>0$ (as in the proof of proposition 3.6, $A\left[\left[Y_{1}, \ldots, Y_{d^{\prime}}\right]\right] /\left(Y_{1} \cdots Y_{r^{\prime}}\right)$ is graded since $\left.t^{\prime}=0\right)$. By proposition $3.6 c_{i} \in(t)$, and hence since we are assuming that $t^{2}=0$ we have $\prod_{i=1}^{r} c_{i}=0$. Thus

$$
t=\prod_{i=1}^{r} \phi\left(X_{i}\right)=\prod c_{i}+g=g
$$

where $g \in\left(Y_{1}, \ldots, Y_{d^{\prime}}\right)$. If $t \neq 0$ this is a contradiction since

$$
A \cap\left(Y_{1}, \ldots, Y_{d^{\prime}}\right)=\{0\} .
$$

Thus there exists an automorphism $\sigma$ of $\mathcal{M}_{T}$ such that the decompositions of $\mathcal{M}_{T}$ obtained from

$$
f^{*} \mathcal{M}_{T} \rightarrow \mathcal{M}_{X}
$$

and

$$
f^{*} \mathcal{M}_{T} \stackrel{\sigma}{\longrightarrow} f^{*} \mathcal{M}_{T} \longrightarrow \mathcal{M}_{X}^{\prime}
$$

are the same. Decompose

$$
\mathcal{M}_{X} \simeq \oplus_{\gamma \in \Gamma, \mathcal{O}_{X}^{*}} \mathcal{M}_{X}^{\gamma}, \quad \mathcal{M}_{X}^{\prime} \simeq \oplus_{\gamma \in \Gamma, \mathcal{O}_{X}^{*}} \mathcal{M}_{X}^{\gamma \prime}
$$

Then to prove the theorem it suffices to show that there exists a unique automorphism $\sigma: \mathcal{M}_{T}^{\gamma} \rightarrow \mathcal{M}_{T}^{\gamma}$ and an isomorphism $\psi: \mathcal{M}_{X}^{\gamma} \simeq \mathcal{M}_{X}^{\gamma \prime}$ such that the diagram

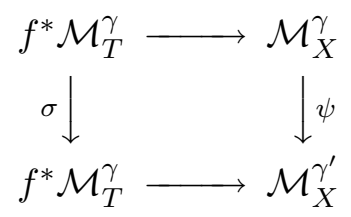

commutes. For this we may as in the proof of theorem 4.1 replace $\underline{X}$ by an open set $\underline{U}$ which contains the component $\gamma$ but does not intersect any of the components $\Gamma-\{\gamma\}$, and we can also choose a chart

$$
\mathbb{N} \rightarrow \mathcal{M}_{T}^{\gamma}
$$

Let $t_{\gamma} \in A$ be the image of 1 . Then using the notation of the proof of theorem 4.1, we want to show that the natural map

$$
\left\{u \in A^{*} \mid u t_{\gamma}=t_{\gamma}\right\} \longrightarrow H^{0}\left(\underline{U}, G_{2} / G\right)
$$

is bijective. This follows from the exactness of the first line in equation 4.6 and corollary 4.3 . 
In light of the uniqueness of $(\sigma, \phi)$, to prove theorem 5.1 it suffices to prove the existence of $(\sigma, \phi)$ étale locally. If $\underline{T}$ is the spectrum of an artinian local ring, then it follows from lemma 5.3 that $\mathcal{M}_{T}$ and $\mathcal{M}_{T}^{\prime}$ are étale locally isomorphic, and hence by proposition 5.2 a pair $(\sigma, \phi)$ exists in this case. By passage to the limit using theorem 4.1 a pair $(\sigma, \phi)$ also exists in the case when $\underline{T}$ is the spectrum of a complete noetherian local ring.

For general $T$, let $t \in \underline{T}$ be a point. Then from above there exists a pair $(\sigma, \phi)$ over the completion $\widehat{\mathcal{O}}_{T, t}$. By the Artin approximation theorem ([3], 1.6), there exists a pointed $\underline{T}$-scheme $v \in \underline{V}$ together with an isomorphism $\widehat{\mathcal{O}}_{\underline{V}, v} \simeq \widehat{\mathcal{O}}_{T, t}$ and a pair $\left(\sigma_{V}, \phi_{V}\right)$ over $\underline{V}$ inducing the pair $(\sigma, \phi)$ over $\widehat{\mathcal{O}}_{T, t}$. By construction $\underline{V} / \underline{T}$ is étale at $v$, and hence after shrinking $\underline{V}$ we can assume that $V$ is an étale $T$-scheme.

This concludes the proof of theorem 5.1.

Theorem 5.4. IVLS admits a smooth cover.

Proof. It suffices to show that for any map

$$
\underline{T} \longrightarrow \log _{\left(\underline{S}, \mathcal{O}_{S}^{*}\right)}
$$

the product

$$
\underline{T} \times \log _{\left(\underline{S}, \mathcal{O}_{S}^{*}\right)} \underline{I V L S}
$$

is representable by an algebraic space. If $\mathcal{M}_{T}$ is the log structure corresponding to the map 5.3, then the product is the functor

$$
S S:(\underline{T}-\text { schemes }) \longrightarrow S e t
$$

which to any $h: \underline{T}^{\prime} \rightarrow \underline{T}$ associates the set of special elements in

$$
\operatorname{IVLS}\left(\left(\underline{T}^{\prime}, h^{*} \mathcal{M}_{T}\right)\right) .
$$

We prove that $S S$ is representable by verifying the conditions of $([5], 5.4)$. For a $\underline{T}$-scheme $h: \underline{T^{\prime}} \rightarrow \underline{T}$ denote by $\left.\mathcal{M}_{T}\right|_{\underline{T}^{\prime}}$ the pullback $h^{*} \mathcal{M}_{T}$ and let $\underline{X}_{T^{\prime}}$ denote the product $\underline{T}^{\prime} \times_{\underline{T}} \underline{X}$.

By proposition 5.2, $S S$ is a sheaf for the étale topology, and $S S$ is limit preserving by $([16], 4.3)$.

Lemma 5.5. Suppose $A^{\prime} \rightarrow A$ is a surjective morphism of rings with squarezero kernel, and suppose $\mathcal{M}_{X_{A}} \in S S(\operatorname{Spec}(A))$. Then to give a lifting of $\mathcal{M}_{X_{A}}$ to $S S\left(\operatorname{Spec}\left(A^{\prime}\right)\right)$ is equivalent to giving a 1-morphism

$$
\mathcal{M}_{X_{A^{\prime}}}: \underline{X}_{A^{\prime}} \longrightarrow \mathcal{L}_{\log }\left(\operatorname{Spec}\left(A^{\prime}\right),\left.\mathcal{M}_{T}\right|_{A^{\prime}}\right)
$$

filling in the diagram 


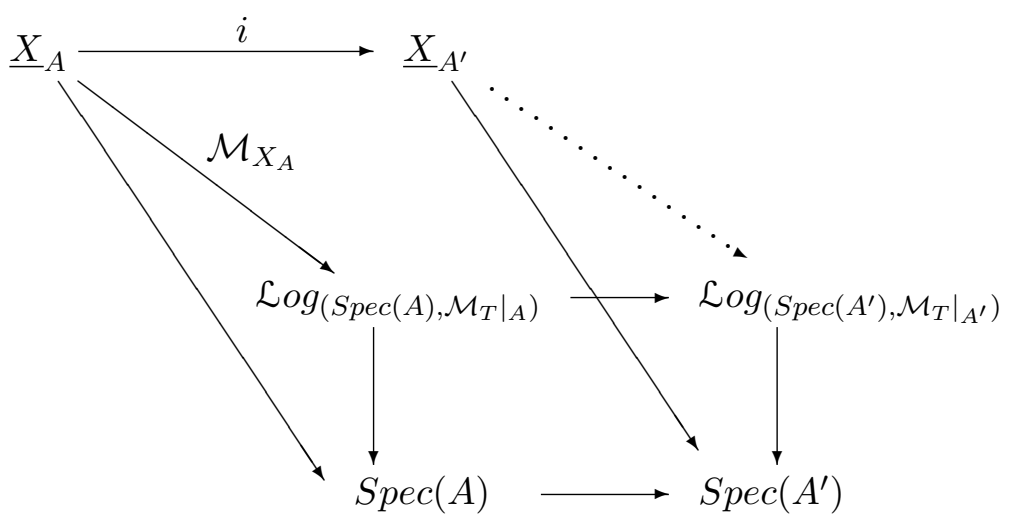

(that is, a 1-morphism $\mathcal{M}_{X_{A^{\prime}}}$ together with an isomorphism $i^{*} \mathcal{M}_{X_{A^{\prime}}} \simeq$ $\left.\mathcal{M}_{X_{A}}\right)$.

Proof. To give a 1-morphism as in the lemma is equivalent to giving a lifting of $\mathcal{M}_{X_{A}}$ to $\underline{X}_{A^{\prime}}$ and a morphism of $\log$ schemes $\left(\underline{X}_{A^{\prime}}, \mathcal{M}_{X_{A^{\prime}}}\right) \rightarrow$ $\left(\operatorname{Spec}\left(A^{\prime}\right),\left.\mathcal{M}_{T}\right|_{A^{\prime}}\right)$ lifting the map from $\left(\underline{X}_{A}, \mathcal{M}_{A}\right)$. Thus the lemma is equivalent to the statement that any such lifting is a special morphism. Now by ([12] 4.1 and [16] theorem 4.4)), any such lifting $\mathcal{M}_{A^{\prime}}$ factors through the maximal open substack $\mathcal{U} \subset \mathcal{L}_{\operatorname{sog}}\left(\operatorname{Spec}\left(A^{\prime}\right),\left.\mathcal{M}_{T}\right|_{A^{\prime}}\right)$ flat over $\operatorname{Spec}\left(A^{\prime}\right)$. Since $\underline{X}_{A^{\prime}} / A^{\prime}$ is flat, this implies that $\mathcal{M}_{X_{A^{\prime}}}$ is smooth since its reduction to $A$ is smooth. The other conditions in the definition of a special morphism evidently only depend on the reduced structure of the base; hence the lemma.

It follows from the lemma and ([17]) that Schlessinger's condition $\left(\mathrm{S}^{\prime}\right)$ holds, and that for any $A$-module $I$, the module $S S_{\mathcal{M}_{X_{A}}}(A[I])$ is isomorphic to

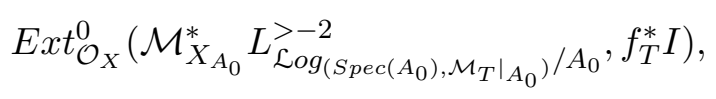

where $L_{\mathcal{L}}^{>-2} \log _{\left(\operatorname{Spec}\left(A_{0}\right),\left.\mathcal{M}_{T}\right|_{A_{0}}\right) / A_{0}}$ denotes the truncated cotangent complex of the algebraic stack $\mathcal{L}_{\log }\left(\operatorname{Spec}\left(A_{0}\right),\left.\mathcal{M}_{T}\right|_{A_{0}}\right)$ (see [17] or [13]). Moreover, ([17]) shows that the modules

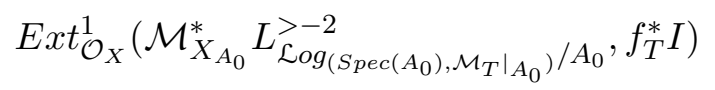

define an obstruction theory for $S S$. To see that the conditions on $D$ and $\mathcal{O}$ of ([5], (S2) and 4.1) are satisfied use ([6], III.3.1.2 and III.4.5) and ([3], lemmas 6.8 and 6.9).

Next we claim that if $A_{0}$ is a reduced ring, and if $\mathcal{M}_{X_{A_{0}}}$ and $\mathcal{M}_{X_{A_{0}}}^{\prime}$ are two elements of $S S\left(\operatorname{Spec}\left(A_{0}\right)\right)$ which are equal at a dense set of points of finite type, then they are equal on a dense open set. To see this observe that by theorem 5.1 there exists a unique pair of isomorphisms $\sigma:\left.\left.\mathcal{M}_{T}\right|_{A_{0}} \rightarrow \mathcal{M}_{T}\right|_{A_{0}}$ 
and $\phi: \mathcal{M}_{X_{A_{0}}} \rightarrow \mathcal{M}_{X_{A_{0}}}^{\prime}$ such that the diagram

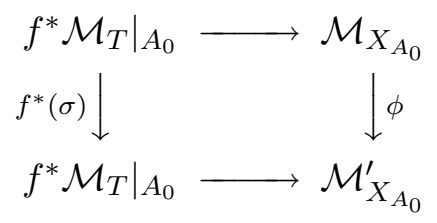

commutes. The condition that $\mathcal{M}_{X_{A_{0}}}$ and $\mathcal{M}_{X_{A_{0}}}^{\prime}$ define the same element of $S S$ is therefore the same as the condition that $\sigma=i d$. Now since $\mathcal{L}_{\left.g_{(\underline{T}}, \mathcal{O}_{T}^{*}\right)}$ is algebraic, if $\sigma=i d$ for a dense set of points of $\operatorname{Spec}\left(A_{0}\right)$ of finite type, then $\sigma$ equals the identity on some dense open set by ([5], page 182).

Finally observe that if $\hat{A}$ is a complete local ring with residue field of finite type over $\underline{T}$, then the map

$$
S S(\hat{A}) \longrightarrow \lim _{\longleftarrow} S S\left(\hat{A} / \mathfrak{m}_{\hat{A}}^{n}\right)
$$

is bijective by theorem 4.1. It therefore follows from $([5], 5.4)$ that $S S$ is representable by an algebraic space over $\underline{T}$. This completes the proof of theorem 5.4 .

To complete the proof of theorem 1.2 , it remains only to see that $\underline{I V L S}$ is of finite type over $\underline{S}$. For this it suffices by the argument given in ([3], page 59) to show the following: given an integral domain $A_{0}$, and a dense set of points $\mathfrak{S} \subset \operatorname{Spec}\left(A_{0}\right)$ of finite type such that $\operatorname{IVLS}(\operatorname{Spec}(k(p))$ is non-empty for all $p \in \mathfrak{S}$, there exists a dense open set $\underline{U} \subset \operatorname{Spec}\left(A_{0}\right)$ for which $\underline{\operatorname{IVLS}}(\underline{U})$ is non-empty. Let $K_{0}=\operatorname{Frac}\left(A_{0}\right)$. Then by assumption, $X_{K_{0}}$ is étale locally isomorphic to

$$
K_{0}\left[X_{1}, \ldots, X_{d}\right] /\left(X_{1} \cdots X_{r}\right),
$$

and so we can replace $A_{0}$ by an affine open set so that $X_{A_{0}}$ is étale locally isomorphic to

$$
A_{0}\left[X_{1}, \ldots, X_{d}\right] /\left(X_{1} \cdots X_{r}\right) .
$$

Let $\mathcal{M}_{A_{0}}$ be the $\log$ structure on $\operatorname{Spec}\left(A_{0}\right)$ associated to the map $\mathbb{N} \rightarrow A_{0}$, $1 \mapsto 0$. By theorem 2.6, to prove the $\underline{I V L S}$ is non-empty over some dense open set of $\operatorname{Spec}\left(A_{0}\right)$, it suffices to prove that there exists a 0 -semi-stable $\log$ structure (in the sense of section 3) on $\underline{X}_{A_{0}}$.

By theorem 3.6 there exists such a log structure if and only if the invertible $\mathcal{O}_{D}$-module

$$
\mathcal{E} x t^{1}\left(\Omega_{\underline{X}_{A_{0}} / A_{0}}^{1}, \mathcal{O}_{X}\right)
$$

is trivial (where $D$ is defined as in section 3). By ([3], page 70) this holds over some dense open set in $\operatorname{Spec}\left(A_{0}\right)$, and hence there exists a dense open set $\underline{U} \subset \operatorname{Spec}\left(A_{0}\right)$ for which $\underline{I V L S}(\underline{U})$ is non-empty. This completes the proof of theorem 1.2. 


\section{REFERENCES}

1. A. Altman and S. Kleiman, Introduction to Grothendieck duality theory, SpringerVerlag, Berlin, 1970, Lecture Notes in Mathematics, Vol. 146. MR 43 \#224

2. M. Artin, Algebraic approximation of structures over complete local rings, Inst. Hautes Études Sci. Publ. Math. (1969), no. 36, 23-58. MR 41 \#6850

3. Algebraization of formal moduli. I, Global Analysis (Papers in Honor of K. Kodaira), Univ. Tokyo Press, Tokyo, 1969, pp. 21-71. MR 41 \#5369

4. _ - The implicit function theorem in algebraic geometry, Algebraic Geometry (Internat. Colloq., Tata Inst. Fund. Res., Bombay, 1968), Oxford Univ. Press, London, 1969, pp. 13-34. MR 41 \#6847

5. _ Versal deformations and algebraic stacks, Invent. Math. 27 (1974), 165-189. MR 53 \#2945

6. J. Dieudonné and A. Grothendieck, Éléments de géométrie algébrique, Inst. Hautes Études Sci. Publ. Math. (1961-1967), no. 4, 8, 11, 17, 20, 24, 28, 32.

7. J. Giraud, Cohomologie non abélienne, Springer-Verlag, Berlin, 1971, Die Grundlehren der mathematischen Wissenschaften, Band 179. MR 49 \#8992

8. R. Hartshorne, Algebraic geometry, Springer-Verlag, New York, 1977, Graduate Texts in Mathematics, No. 52. MR 57 \#3116

9. L. Illusie and A. Ogus, Lectures on logarithmic algebraic geometry, manuscript notes, 2000.

10. F. Kato, Log smooth deformation theory, Tôhoku Math. J. (2) 48 (1996), no. 3, 317354. MR 99a:14012

11. Log smooth deformation and moduli of log smooth curves, Internat. J. Math. 11 (2000), no. 2, 215-232. MR 1754621

12. K. Kato, Logarithmic structures of Fontaine-Illusie, Algebraic analysis, geometry, and number theory (Baltimore, MD, 1988), Johns Hopkins Univ. Press, Baltimore, MD, 1989, pp. 191-224. MR 99b:14020

13. G. Laumon and L. Moret-Bailly, Champs algébriques, Springer-Verlag, Berlin, 2000. MR 1771927

14. J. S. Milne, Étale cohomology, Princeton University Press, Princeton, N.J., 1980. MR 81j:14002

15. A. Ogus, Logarithmic de Rham cohomology, preprint, 1999.

16. M. Olsson, Logarithmic geometry via algebraic stacks, submitted for publication.

17. _ On the deformation theory of algebraic stacks, in preparation.

Department of Mathematics, Massachusetts Institute of Technology, BuildIng 2, 77 Massachusetts Avenue, Cambridge, MA 02139-4307, USA.

E-mail address: molsson@math.mit.edu 